WUB/12-04

\title{
Fermions as Global Correction: the QCD Case
}

\author{
Jacob Finkenrath $^{a}$, Francesco Knechtli ${ }^{a}$, Björn Leder ${ }^{a, b}$ \\ ${ }^{a}$ Department of Physics, Bergische Universität Wuppertal \\ Gaussstr. 20, D-42119 Wuppertal, Germany \\ ${ }^{b}$ Department of Mathematics, Bergische Universität Wuppertal \\ Gaussstr. 20, D-42119 Wuppertal, Germany
}

\begin{abstract}
It is widely believed that the fermion determinant cannot be treated in global acceptance-rejection steps of gauge link configurations that differ in a large fraction of the links. However, for exact factorizations of the determinant that separate the ultraviolet from the infrared modes of the Dirac operator it is known that the latter show less variation under changes of the gauge field compared to the former. Using a factorization based on recursive domain decomposition allows for a hierarchical algorithm that starts with pure gauge updates of the links within the domains and ends after a number of filters with a global acceptance-rejection step. Ratios of determinants have to be treated stochastically and we construct techniques to reduce the noise. We find that the global acceptance rate is high on moderate lattice sizes and demonstrate the effectiveness of the hierarchical filter.
\end{abstract}




\section{Introduction}

The state of the art simulation algorithm for lattice QCD is the Hybrid Monte Carlo (HMC) [1, 2]. As the continuum limit is approached, when the lattice spacing $a$ goes to zero, the simulation cost for a given observable scales typically as $a^{-(5+z)}$. The dynamical critical exponent $z$ depends on the observable and is responsible for the critical slowing down of the simulations. Recently in [3] it was shown that $z\left(Q^{2}\right)=5$ for the topological charge $Q$ (the scaling might even be exponential in $1 / a$ cf. [4]). This is a common problem for all present algorithms for gauge theories and the reason has been traced back to the fact that simulations on periodic lattices get stuck in topological sectors [5]. In fact, on lattices with open boundary conditions $z\left(Q^{2}\right)=2$ is found in [6]. Our original motivation was to look for an alternative algorithm which allows for larger steps in the space of gauge fields.

In recent years new actions to simulate QCD on the lattice have been developed, in particular, based on smearing of the gauge links in the Dirac operator. In the case of Wilson fermions the stability of the HMC algorithm is influenced by the fluctuations of the smallest eigenvalues of the Wilson-Dirac operator [7]. The results of [8] show evidence that smearing improves the stability. The HMC requires the computation of forces (i.e. derivatives of the Dirac operator with respect to the gauge links) and this can be very complicated or even impossible, like when HYP smearing [9] is used. A number of solutions exist, like using stout [10, nHYP [9] or HEX [8, 11] smearing or a differentiable approximation to the $\mathrm{SU}(3)$ projection for the smeared links [12], but flexibility in the choice of gauge and fermion actions is highly desirable and so the question arises, whether an alternative algorithm without force computations exists.

In this article we study, motivated by a previous work in the Schwinger model [13], an algorithm based on global acceptance-rejection steps accounting for the fermion determinant in QCD with $N_{\mathrm{f}}=2$ quark flavors. The basic idea is to make a gauge proposal which is accepted with a probability that depends on the ratio of fermion determinants on the "new" and "old" gauge configurations. Such an algorithm has already been used in QCD simulations with HYP-smeared link staggered fermions [14 16], with the fixed point action [17] and in [18]. The problem with this type of algorithms is their scaling with the lattice volume $V, 1$ The cost of an exact determinant computation grows with $V^{3}$ and the acceptance to change a finite fraction of links decreases like $\exp (-V)$.

In order to avoid the computation of exact determinants we use a stochas-

\footnotetext{
${ }^{1}$ Unless otherwise specified, in this article we use lattice units (i.e. we set $a=1$ ) and $V$ is the number of lattice points.
} 
tic estimation. This estimation can naively introduce a noise which grows like $\exp (V)$. In order to tackle these problems we construct a hierarchical filter of acceptance-rejection steps which successively filters the large fluctuations of the gauge proposal [19]. Hierarchical acceptance-rejection steps based on approximations of the determinant with increasing accuracy were introduced and tested in 20]. Here the filter relies on an exact factorization of the fermion determinant based on domain decomposition [21], which separates the short distance from the long distance scales of the lattice. A hierarchy of block acceptance-rejection steps was proposed in [22] but has never been tested.

The article is organized as follows. In Section 2 we introduce the hierarchical filter of acceptance-rejection steps. Its construction based on domain decomposition is detailed in Section 3. The techniques we use for the stochastic estimation of determinant ratios are presented in Section 4. In particular we introduce an interpolation of the gauge fields which also allows to compute the exact (i.e. without stochastic noise) acceptance. Results for the latter and the effectiveness of the filter are shown in Section [5. Section [6 presents simulation results of $16^{4}$ and $32 \times 16^{3}$ lattices using a filter with three acceptance-rejection steps. A comparison with the HMC is made for observables like the plaquette or the topological charge. In the conclusions Section 7 we also discuss the scaling with the volume. Appendix A contains the proof of detailed balance, Appendix B describes the technique of relative gauge fixing used for the stochastic estimation and Appendix C explains how the acceptance is enhanced by the use of additional parameters.

\section{Hierarchy of acceptance steps}

Let $P(s)$ be the desired distribution of the states $s$ of a system. Suppose a process that proposes a new state $s^{\prime}$ with transition probability $T_{0}\left(s \rightarrow s^{\prime}\right)$ and fulfills detailed balance with respect to $P_{0}(s)$. A process with fixed point distribution $P(s)$ is then obtained by the combination of such a proposal with a subsequent Metropolis acceptance-rejection step [23]

$$
\begin{aligned}
& \text { 0) Propose } s^{\prime} \text { according to } T_{0}\left(s \rightarrow s^{\prime}\right) \\
& \text { 1) } \quad P_{\text {acc }}\left(s \rightarrow s^{\prime}\right)=\min \left\{1, \frac{P_{0}(s) P\left(s^{\prime}\right)}{P(s) P_{0}\left(s^{\prime}\right)}\right\} .
\end{aligned}
$$

This hierarchy of a proposal step and an acceptance-rejection step can easily be generalized to an arbitrary number of acceptance-rejection steps. The result of the first acceptance-rejection step 1) is then interpreted as the proposal for a second acceptance-rejection step 2) and so on. If the target distribution $P(s)$ factorizes 
into $n+1$ parts

$$
P(s)=P_{0}(s) P_{1}(s) P_{2}(s) \ldots P_{n}(s),
$$

the resulting hierarchical acceptance-rejection steps take the form

0) Propose $s^{\prime}$ according to $T_{0}\left(s \rightarrow s^{\prime}\right)$

1) $P_{\text {acc }}^{(1)}\left(s \rightarrow s^{\prime}\right)=\min \left\{1, \frac{P_{1}\left(s^{\prime}\right)}{P_{1}(s)}\right\}$

2) $P_{\text {acc }}^{(2)}\left(s \rightarrow s^{\prime}\right)=\min \left\{1, \frac{P_{2}\left(s^{\prime}\right)}{P_{2}(s)}\right\}$

n) $\quad P_{\text {acc }}^{(n)}\left(s \rightarrow s^{\prime}\right)=\min \left\{1, \frac{P_{n}\left(s^{\prime}\right)}{P_{n}(s)}\right\}$.

In the context of lattice QCD it is plausible to assume $P_{i}(s) \propto \exp \left(-S_{i}(s)\right)$ with real actions $S_{i}$ and thus

$$
\frac{P_{i}\left(s^{\prime}\right)}{P_{i}(s)}=\mathrm{e}^{-\Delta_{i}\left(s, s^{\prime}\right)},
$$

where $\Delta_{i}\left(s, s^{\prime}\right)=S_{i}\left(s^{\prime}\right)-S_{i}(s)$. The average acceptance rate in step $\left.i\right)$ is defined by

$$
\left\langle P_{\mathrm{acc}}^{(i)}\right\rangle_{s, s^{\prime}}=\sum_{s} P(s) \sum_{s^{\prime}} P_{0}\left(s^{\prime}\right) P_{1}\left(s^{\prime}\right) \ldots P_{i-1}\left(s^{\prime}\right) \min \left\{1, \mathrm{e}^{-\Delta_{i}\left(s, s^{\prime}\right)}\right\}
$$

It can be computed assuming a Gaussian distribution for $\Delta_{i}\left(s, s^{\prime}\right)$ with variance $\Sigma_{i}^{2}$ and the result is [13] (see also [24])

$$
\left\langle P_{\mathrm{acc}}^{(i)}\right\rangle_{s, s^{\prime}}=\operatorname{erfc}\left(\sqrt{\Sigma_{i}^{2} / 8}\right) .
$$

The acceptance rates might be enhanced by parameterizing and tuning the factorization (2.2), see Appendix C.

Our goal is to simulate QCD with $N_{\mathrm{f}}=2$ mass-degenerate fermions. After integration over the Grassmann fermion fields the states $s$ are defined by the gauge field $U$ and the target probability distribution is

$$
P(U)=\frac{|\operatorname{det}(D(U))|^{2} \mathrm{e}^{-S_{g}(U)}}{Z},
$$

where $S_{g}$ is the gauge action, $D$ is the lattice Dirac operator and $Z$ is the partition function

$$
Z=\int D[U]|\operatorname{det} D(U)|^{2} \mathrm{e}^{-S_{g}(U)}
$$


The integration measure is $D[U]=\prod_{x, \mu} d U(x, \mu)$, where $d U(x, \mu)$ is the $\mathrm{SU}(3)$ Haar measure for the link $U(x, \mu)$.

A simple two-step algorithm would consist of some update of the gauge link configuration $U \rightarrow U^{\prime}$, which fulfills detailed balance with respect to $P_{0}(U) \propto$ $\exp \left(-S_{g}(U)\right)$, followed by an acceptance-rejection step with the fermion determinant ratio

$$
P_{\text {acc }}^{(1)}\left(U \rightarrow U^{\prime}\right)=\min \left\{1, \operatorname{det} \frac{D\left(U^{\prime}\right)^{\dagger} D\left(U^{\prime}\right)}{D(U)^{\dagger} D(U)}\right\} .
$$

The proof of detailed balance can be found in Section A.1.

If the proposal changes only one link and the Dirac operator $D$ is ultra-local it is easy to show that the acceptance-rejection step requires only few inversions. 2 . An ergodic algorithm is then obtained by sweeps through the lattice. Thus the cost of such an algorithm would scale with the lattice volume $V$ at least like $V^{2}$ [25] and it requires $\mathrm{O}(V)$ inversions per sweep.

If, on the other hand, a finite fraction $\propto V$ of the links is updated for the proposal, the acceptance rate decreases exponentially with the volume. In order to see this we write the distribution $P_{1}$ as $P_{1}(U) \propto \exp \left(\ln \left(\operatorname{det} D^{\dagger} D\right)\right)$. The action difference $\Delta_{1}\left(U, U^{\prime}\right)=\ln \left(\operatorname{det} D\left(U^{\prime}\right)^{\dagger} D\left(U^{\prime}\right)\right)-\ln \left(\operatorname{det} D^{\dagger}(U) D(U)\right)$ can be written as $\Delta_{1}=-\sum_{i} \ln \left(\lambda_{i}\right)$ in terms of the eigenvalues $\lambda$ of the operator $M^{\dagger} M$ with

$$
M=D\left(U^{\prime}\right)^{-1} D(U)
$$

If we assume a Gaussian distribution 3 (after averaging over the gauge ensemble $U$ and the proposals $\left.U^{\prime}\right)$ for the logarithms of the eigenvalues $\hat{\lambda}_{i}=\ln \left(\lambda_{i}\right)$ with mean zero and variance $\sigma_{\hat{\lambda}}^{2}$, we can approximate

$$
\Sigma_{1}^{2} \approx \bar{N}_{1} \sigma_{\hat{\lambda}}^{2} / 2
$$

where $\bar{N}_{1}$ is the number of eigenvalues $\lambda \neq 1$. Typically $\bar{N}_{1} \propto V$ and this implies that $\Sigma_{1}^{2}$ is proportional to the volume $V$. The complementary error function in the formula for the acceptance (2.6) has the asymptotic expansion $\operatorname{erfc}(x) \sim$ $\exp \left(-x^{2}\right) /(x \sqrt{\pi})\left(1-1 /\left(2 x^{2}\right)+\cdots\right)$ for $|x| \gg 1$ which shows the exponential decrease with the volume.

From the preceding discussion it is obvious that such two-step algorithms will not be efficient for large lattices. Indeed numerical experiments show that for lattices larger than $\sim(0.2 \mathrm{fm})^{4}$ (where all links are updated) the acceptance rate quickly becomes less than a percent. However, in the context of low mode

\footnotetext{
${ }^{2}$ For example, in the case of the Wilson-Dirac operator 12 inversions are needed.

3 We verified numerically that this assumption is valid to a good approximation.
} 
reweighting the fluctuations of the determinant of $D_{\text {low }}^{\dagger} D_{\text {low }}$, where $D_{\text {low }}$ is a restriction of $D$ to its low modes, are found to depend only mildly on the volume [26]. The explanation for this observation might be the fact that the width of the distribution of the small eigenvalues of $\sqrt{D^{\dagger} D}$ decrease like $1 / V$ [26] (the fluctuations of the eigenvalue gap go instead like $1 / \sqrt{V}[7]$ ). Thus, given a factorization of the determinant that separates low (infrared IR) and high (ultraviolet UV) modes

$$
\operatorname{det}(D)=\operatorname{det}\left(D_{\mathrm{UV}}\right) \cdots \operatorname{det}\left(D_{\mathrm{IR}}\right)
$$

a hierarchy of acceptance steps can be constructed, where the large fluctuations of the UV modes go through a set of filters (acceptance-rejection steps) which are more and more dominated by the IR modes:

$\begin{array}{cccccc}\text { 0) } & P_{0} & \text { UV } & \text { short distance } & \text { local } & \text { cheap } \\ & \vdots & \vdots & \vdots & \vdots & \vdots \\ n) & P_{n} & \text { IR } & \text { long distance } & \text { global } & \text { expensive }\end{array}$

This hierarchy of modes may induce also a hierarchy of costs since it is the low modes that cause the most cost in lattice QCD. Furthermore the factorization should be exact and the terms simple to compute. Factorizations that realize these conditions are already used to speed-up the HMC algorithm, i.e., in the context of mass-preconditioning [27] and domain decomposition [21]. Only the latter also allows for a decoupling of local updates and will be discussed in the following.

\section{Domain decomposition}

Domain decomposition was introduced in lattice QCD in [22] and in [21] the resulting factorization of the fermion determinant was used to separate short distance and long distance physics in the HMC algorithm. For definiteness we consider here the Wilson-Dirac operator $D(U)$ [28], which may include the clover term needed for $\mathrm{O}(a)$ improvement [29, 30]. But our algorithm is applicable to a more general class of Dirac operators, see below.

Suppose a decomposition $\mathcal{C}$ of the lattice in non-overlapping blocks $b \in \mathcal{C}$ (cf. Fig. 1 for a 2-dimensional visualization). The lattice sites are labeled such that the sites belonging to the first black block come first, then the second black block and after the last black block the first white block and so on. The Dirac operator can then be written as

$$
D=\left(\begin{array}{cc}
D_{\mathrm{bb}} & D_{\mathrm{bw}} \\
D_{\mathrm{wb}} & D_{\mathrm{ww}}
\end{array}\right),
$$




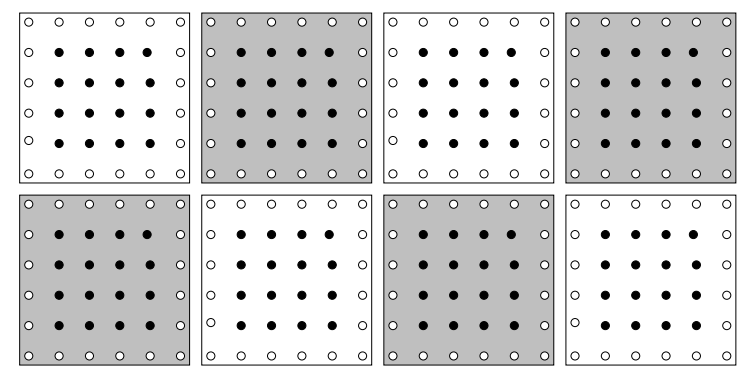

Figure 1: Block decomposition of a 2-dimensional lattice. The blocks are coloured like a checker board. Picture taken from [21].

where $D_{\mathrm{bb}}\left(D_{\mathrm{ww}}\right)$ is a block-diagonal matrix with the black (white) block Dirac operators $D_{b}$ on the diagonal. The block Dirac operators $D_{b}$ fulfill Dirichlet boundary conditions and therefore are dominated by short distance physics (if the blocks are small enough). The matrices $D_{\mathrm{bw}}$ and $D_{\mathrm{wb}}$ contain the block interaction terms. The form (3.1) induces a factorization of the determinant

$$
\operatorname{det}(D)=\prod_{b \in \mathcal{C}} \operatorname{det}\left(D_{b}\right) \operatorname{det}(\hat{D}), \quad \hat{D}=1-D_{\mathrm{bb}}^{-1} D_{\mathrm{bw}} D_{\mathrm{ww}}^{-1} D_{\mathrm{wb}}
$$

where $\hat{D}$ is the Schur complement of the decomposition (3.1) and contains block interactions, i.e. the long distance physics. A natural separation scale is given by the inverse block size $1 / L_{b}$. In the context of the domain decompositioned HMC the average force associated with the Schur complement is an order of magnitude smaller than the force associated with the block Dirac operators [21]. This indicates that the fluctuations of the determinant of the Schur complement are smaller than that of the block determinants. Furthermore the factorization (3.2) can be iterated using a recursive domain decomposition

$$
\operatorname{det}\left(D_{b}\right)=\prod_{b^{\prime} \in \mathcal{C}_{b}} \operatorname{det}\left(D_{b^{\prime}}\right) \operatorname{det}\left(\hat{D}_{b}\right)
$$

We note that the Schur complement $\hat{D}_{b}$ fulfills Dirichlet boundary conditions. We have implemented the recursive domain decomposition in the freely available software package DD-HMC by M. Lüscher [31]. In the case of one level of recursion 
the hierarchy of acceptance-rejection steps is given by

$$
\begin{aligned}
& \text { 1) } P_{\text {acc }}^{(1)}=\min \left\{1, \operatorname{det} \frac{D_{b^{\prime}}\left(U^{\prime}\right)^{\dagger} D_{b^{\prime}}\left(U^{\prime}\right)}{D_{b^{\prime}}(U)^{\dagger} D_{b^{\prime}}(U)}\right\}, \quad \forall b, \forall b^{\prime} \in \mathcal{C}_{b} \\
& \text { 2) } P_{\text {acc }}^{(2)}=\min \left\{1, \operatorname{det} \frac{\hat{D}_{b}\left(U^{\prime}\right)^{\dagger} \hat{D}_{b}\left(U^{\prime}\right)}{\hat{D}_{b}(U)^{\dagger} \hat{D}_{b}(U)}\right\}, \quad \forall b \in \mathcal{C} \\
& \text { 3) } P_{\text {acc }}^{(3)}=\min \left\{1, \operatorname{det} \frac{\hat{D}\left(U^{\prime}\right)^{\dagger} \hat{D}\left(U^{\prime}\right)}{\hat{D}(U)^{\dagger} \hat{D}(U)}\right\} .
\end{aligned}
$$

At the beginning the set of links to be updated, the so called active links, is chosen such that the acceptance-rejection steps for the smallest blocks, $b^{\prime}$, at stage 1) in Eq. (3.4) decouple and can therefore be processed in parallel. In the case of Wilson fermions with or without clover term the active links are the links that have at most one endpoint on the boundary of a block (white points in Fig. 11). In this case the block acceptance steps also decouple if the links in the Wilson-Dirac operator (but not in the clover term) are replaced by one level of HYP smearing [32. After the last and global acceptance-rejection step the gauge field is translated by a random vector, see Appendix $\mathrm{C}$ of [21].

If the smallest blocks, $b^{\prime}$, at stage 1) in Eq. (3.4) consist of no more than $\sim 6^{4}$ lattice points, the determinant ratios can be efficiently computed exactly by LU-decomposition [33]. If the smallest blocks are larger, we compute their determinants by a factorization like in Eq. (3.3). The Schur complements at the stages 2) and 3) in Eq. (3.4) are usually too large for their determinant ratios to be computed exactly and have to be treated stochastically. The stochastic estimation of determinant ratios is the topic of the next section. Following this discussion we give to our algorithm the name of Partially Stochastic Multi-Step (PSMS) algorithm.

\section{Stochastic techniques for determinant ratios}

Since the numerical cost for the computation of exact determinants grows with the cube of the size of the matrix, determinants of Dirac operators for lattices larger than $6^{4}$ have to be estimated stochastically. In particular for our problem we have to estimate ratios of determinants of Schur complements, which arise from a domain decomposition and appear in the acceptance-rejection steps of Eq. (3.4). In Appendix A we show that such stochastic acceptance-rejection steps

\footnotetext{
${ }^{4}$ The same applies to Schur complements arising from a factorization of the smallest blocks, if that is needed.
} 
fulfill detailed balance. In this section we describe in detail the techniques we use to reduce the associated stochastic noise.

In Section 4.1 we discuss the stochastic noise introduced when the determinant ratio in Eq. (2.9) (for generic Dirac operators $D$ ) is evaluated stochastically. The stochastic noise depends on the spectrum of generalized eigenvalues of the operators forming the ratio [13. In order to reduce it we apply techniques described in Section 4.2 and Section 4.3. In Section 4.2 we discuss a relative gauge fixing of the gauge field $U$ and $U^{\prime}$. This gauge fixing is applied for the construction of a gauge field interpolation, a new method which we present in Section 4.3. The gauge fields are linearly interpolated and this induces a factorization in terms of ratios of operators which can be made arbitrarily close as the number of interpolation steps increases. In particular, there exists the limit in which the exact ratio is obtained. In Section 4.4 the properties of the Schur complement are reviewed. In this particular case the noise vector can be restricted to a subspace of the boundary points of the blocks. In Section 4.5 we support the introduction of these techniques by numerical results.

\subsection{Stochastic estimation of determinant ratios}

We replace the determinants of ratios of Dirac operators in Eq. (2.9) by stochastic estimators

$$
\min \left\{1, \operatorname{det}\left(M^{\dagger} M\right)^{-1}\right\} \longrightarrow \min \left\{1, \mathrm{e}^{-|M \eta|^{2}+|\eta|^{2}}\right\}
$$

where the ratio operator $M$ is defined in Eq. (2.10). In Eq. (4.1) $\eta$ is a complex Gaussian noise vector that is updated before each acceptance-rejection step and $|\eta|^{2}$ is its norm squared, see Section A.2. The average over $\eta$ of a function $f(\eta)$ is defined by

$$
\langle f(\eta)\rangle_{\eta}=\int D[\eta] \mathrm{e}^{-|\eta|^{2}} f(\eta)
$$

The measure $D[\eta]$ is normalized such that $\int D[\eta] \exp \left(-|\eta|^{2}\right)=1$. The algorithm satisfies detailed balance (the proof is given in Section A.2) and yields an acceptance rate that is bounded from above by the exact acceptance in Eq. (2.9) [13]. There are other possible choices for the distribution of $\eta$ than a Gaussian distribution. But because of the central limit theorem these other choices are equivalent to the Gaussian distribution in the large volume limit.

The stochastic noise introduced in the acceptance-rejection step by Eq. (4.1) has the effect of replacing in Eq. (2.6)

$$
\Sigma^{2} \longrightarrow \sigma^{2}=\Sigma^{2}+\left(\sigma^{\text {stoch }}\right)^{2}
$$


where

$$
\left(\sigma^{\text {stoch }}\right)^{2}=\left\langle\Delta^{2}\right\rangle_{U, U^{\prime}, \eta}-\left(\langle\Delta\rangle_{U, U^{\prime}, \eta}\right)^{2}
$$

with $\Delta=|M \eta|^{2}-|\eta|^{2}$. The average $\langle\cdot\rangle_{U, U^{\prime}, \eta}$ is taken over the gauge ensemble $U$, the proposals $U^{\prime}$ and the noise vectors $\eta$. For given $U$ and $U^{\prime}$ Eq. (4.4) can be computed by performing the integrations over $\eta$ in the basis of orthonormal eigenvectors of $M^{\dagger} M$ with eigenvalues $\lambda_{k}$, cf. [13]. The result is

$$
\left(\sigma^{\text {stoch }}\right)^{2}=\left\langle\sum_{k}\left(\lambda_{k}-1\right)^{2}\right\rangle_{U, U^{\prime}} .
$$

The eigenvalues $\lambda=1$ do not contribute to the variance. If we denote by $h_{1}$ the full width at half maximum (FWHM) of the distribution of the eigenvalues $\lambda_{k}$ and by $\bar{N}_{1}$ the number of eigenvalues which are not one, we can approximate

$$
\left(\sigma^{\text {stoch }}\right)^{2} \approx \bar{N}_{1} h_{1}^{2}
$$

It becomes clear that the smaller $\bar{N}_{1}$ and $h_{1}$ are, the larger the stochastic acceptance will be. Furthermore in 34 ] it was noted that the spectrum of $M^{\dagger} M$ has to fulfill the condition $\lambda>0.5$, because otherwise the variance of the quantity under the minimum function in Eq. (4.1) is not defined.

\subsection{Relative gauge fixing}

In [13] (see also [17]) it was noticed that relative gauge fixing of the configuration $U$ and $U^{\prime}$ reduces the stochastic noise in Eq. (4.4). Under a gauge transformation $g(x) \in \mathrm{SU}(3)$, the gauge links transform as $U(x, \mu) \rightarrow U^{g}(x, \mu)=$ $g(x) U(x, \mu) g(x+\hat{\mu})^{-1}$ and the Dirac operator as

$$
\left.D\left(U^{g}\right)_{x y}=g(x) D(U)_{x y} g(y)^{-1} \quad \text { (no sum over } x \text { and } y\right),
$$

where we suppress the spin indices. Further we define a scalar product of two gauge fields as

$$
\left(U, U^{\prime}\right)=\frac{1}{12 V} \sum_{x, \mu} \operatorname{Re} \operatorname{Tr}\left\{1-U(x, \mu)^{\dagger} U^{\prime}(x, \mu)\right\} .
$$

Relative gauge fixing is defined through the minimization

$$
\begin{aligned}
& \min _{g_{1}, g_{2}}\left(U^{g_{1}}, U^{\prime g_{2}}\right)=\min _{g_{1}, g_{2}} \frac{1}{12 V} \sum_{x, \mu} \operatorname{Re} \operatorname{Tr}\{ \\
& \left.1-g_{1}(x+\hat{\mu}) U(x, \mu)^{\dagger} g_{1}(x)^{-1} g_{2}(x) U^{\prime}(x, \mu) g_{2}(x+\hat{\mu})^{-1}\right\} .
\end{aligned}
$$

\footnotetext{
5 The eigenvalues $\lambda$ of $M^{\dagger} M$ are equivalent to the generalized eigenvalues of the problem $D(U) D(U)^{\dagger} \chi=\lambda D\left(U^{\prime}\right) D\left(U^{\prime}\right)^{\dagger} \chi$.
} 
We determine $g_{1}$ and $g_{2}$ before the acceptance-rejection step Eq. (4.1), where we use

$$
M=D\left(U^{\prime g_{2}}\right)^{-1} D\left(U^{g_{1}}\right) .
$$

Relative gauge fixing does not change the exact acceptance rates in Eq. (3.4) but in general improves the stochastic acceptance rate in Eq. (4.1). In order to show detailed balance in the latter case, consider the reverse transition $U^{\prime} \rightarrow U$, for which the minimization is $\min _{\tilde{g}_{1}, \tilde{g}_{2}}\left(U^{\prime \tilde{g}_{1}}, U^{\tilde{g}_{2}}\right)$. As one can immediately see by taking the complex conjugate of Eq. (4.9) the result is given by $\tilde{g}_{1}=g_{2}$ and $\tilde{g}_{2}=g_{1}$. This implies for the reverse transition

$$
M \rightarrow \tilde{M}=D\left(U^{\tilde{g}_{2}}\right)^{-1} D\left(U^{\prime \tilde{g}_{1}}\right)=M^{-1}
$$

which is precisely the property needed to prove detailed balance [13].

In the above procedure, the choice of $g_{1}$ and $g_{2}$ is not unique. In fact one can transform $g_{1} \rightarrow g_{1} h$ and $g_{2} \rightarrow g_{2} h$ by some other gauge transformation $h(x)$ and the minimization condition Eq. (4.9) is unchanged. Instead we choose 6

$$
g_{1}=g_{2}^{-1}=g \text {. }
$$

The numerical procedure for the minimization Eq. (4.9) using Eq. (4.12) is described in Appendix B.

In the proposal $U \rightarrow U^{\prime}$ we only change active links in the blocks and we restrict the gauge transformations $g$ in Eq. (4.12) to the black points in Fig. 1 . One reason for this is that the critical slowing down of such a local (i.e. restricted to the blocks) minimization is reduced compared to a global minimization over the entire lattice.

\subsection{Gauge field interpolation}

In order to ensure $\lambda>0.5$ and bring the spectrum of $M^{\dagger} M$ closer to one, one could employ the method of determinant breakup introduced in [20,34]. It uses the factorization $\operatorname{det}\left(M^{\dagger} M\right)=\left[\operatorname{det}\left(\left(M^{\dagger} M\right)^{1 / N}\right)\right]^{N}$ and in the stochastic acceptancerejection step Eq. (4.1) each factor is then replaced by a stochastic estimator with an independent noise vector. The effect on the spectrum of $M^{\dagger} M$ is to replace $\lambda \rightarrow \lambda^{1 / N}$. The gauge field interpolation which we propose in this article has a similar effect but avoids the computation of $1 / N$ th roots of $M^{\dagger} M$.

We introduce a sequence of intermediate fields $U_{i}, i=0, \ldots, N$ which starts from the gauge field $U_{0}=U^{g}$ and ends with the gauge field $U_{N}=U^{\prime g^{-1}} \cdot g$ is the

\footnotetext{
${ }^{6}$ We thank Ulli Wolff for suggesting this choice.
} 
gauge transformation in Eq. (4.12). The determinant of $M^{\dagger} M$ can be factorized like

$$
\operatorname{det}\left(M^{\dagger} M\right)=\prod_{i=0}^{N-1} \operatorname{det}\left(M_{i}^{\dagger} M_{i}\right)
$$

where

$$
M_{i}=D\left(U_{i+1}\right)^{-1} D\left(U_{i}\right) .
$$

The stochastic acceptance-rejection step in Eq. (4.1) is done by drawing one independent Gaussian noise vector $\xi_{i}$ for each factor

$$
\min \left\{1, \mathrm{e}^{\sum_{i=0}^{N-1}-\left|M_{i} \xi_{i}\right|^{2}+\left|\xi_{i}\right|^{2}}\right\} .
$$

The cost is then one inversion for each factor. In order for the algorithm to fulfill detailed balance the intermediate gauge configurations have to be the same when doing the reverse change $U^{\prime} \rightarrow U$. The proof of detailed is given in Section A.3.

The simplest way to construct such an interpolation is

$$
U_{i}(x, \mu)=\frac{N-i}{N} U^{g}(x, \mu)+\frac{i}{N} U^{\prime g^{-1}}(x, \mu), \quad i=0,1, \cdots, N-1,
$$

which interpolates linearly between $U_{0}=U^{g}$ and $U_{N}=U^{g^{-1}}$. The interpolation has no physical meaning, only numerical efficiency counts. The intermediate fields are not $\mathrm{SU}(3)$ matrices, in the Dirac operator we use $U_{i}^{\dagger}$ (and not $U_{i}^{-1}$ ) in order to preserve the $\gamma_{5}$ Hermiticity of the Wilson-Dirac operator. Since $\left\|U_{i}-U_{i+1}\right\| \propto 1 / N, \forall i<N$, we expect the eigenvalues $\lambda_{k}^{(i)}$ of $M_{i}^{\dagger} M_{i}$ to be $\lambda_{k}^{(i)}=1+\mathrm{O}\left(h_{1} / N\right)$ and so the FWHM of their distribution 7 can be approximated by $h_{N} \approx h_{1} / N$ in terms of the FWHM $h_{1}$ of the eigenvalue distribution of $M^{\dagger} M$. The stochastic noise in the acceptance-rejection step is reduced to

$$
\left(\sigma_{N}^{\text {stoch }}\right)^{2} \approx N \bar{N}_{1} h_{N}^{2} \approx \bar{N}_{1} \frac{h_{1}^{2}}{N}
$$

as compared to Eq. (4.6). An important feature of this method is the limit $N \rightarrow \infty$, for which $\sigma_{N}^{\text {stoch }} \rightarrow 0$ and we recover the exact acceptance, cf. Eq. (4.3).

\subsection{Schur Complement}

The Schur complement in Eq. (3.2) is $\hat{D}=1-Q$ with $Q=D_{\mathrm{bb}}^{-1} D_{\mathrm{bw}} D_{\mathrm{ww}}^{-1} D_{\mathrm{wb}}$. Let us denote by $P$ the orthonormal projector to the space of the white points in the

\footnotetext{
${ }^{7}$ In the case of the full Dirac operator, we find numerically that the smallest (largest) eigenvalue change with $N$ as $\lambda_{\min }^{(i)} \sim \exp \{-b / N\}\left(\lambda_{\max }^{(i)} \sim \exp \left\{b^{\prime} / N\right\}\right)$ for positive constants $b$ $\left(b^{\prime}\right)$. This is the same behavior one obtains using the determinant breakup in $1 / N$ th roots.
} 
black blocks in Fig. 1. For the points which have only one nearest neighbor on a different block, $P$ projects to only two of the four spin components. The explicit definition of $P$ can be found in Appendix $B$ of [21. It does not depend on the gauge field and it satisfies the properties $D_{\mathrm{wb}} P=D_{\mathrm{wb}}$ and $P^{2}=P$ which imply

$$
\operatorname{det}(1-Q)=\operatorname{det}(1-P Q) \text {. }
$$

This means that one can use $1-P Q$ instead of $\hat{D}$ in Eq. (4.1) and therefore the noise $\eta$ is defined only on the space invariant under $P$. We also need to apply the inverse of the operator $1-P Q$ which is [21]

$$
(1-P Q)^{-1}=1-P D^{-1} D_{\mathrm{wb}}
$$

Here $D_{\mathrm{wb}}$ is meant to act on the total space of points (by padding with zeros). For a global lattice of sizes $L_{\mu}$ in directions $\mu=0,1,2,3$ and a domain decomposition into blocks of sizes $l_{\mu}$, the dimension of the space invariant under $P$ is

$$
\operatorname{dim}(P)=6 \prod_{\mu=0}^{3} \frac{L_{\mu}}{l_{\mu}}\left(\sum_{\nu=0}^{3} \frac{l_{0} l_{1} l_{2} l_{3}}{l_{\nu}}-4 \sum_{\nu=0}^{3}\left(l_{\nu}-1\right)\right) .
$$

For the number $\bar{N}_{1}$ in Eq. (4.6) we have $\bar{N}_{1} \leq \operatorname{dim}(P)$. On a lattice with the same number of points $L$ in all directions, if we choose $l_{\mu}=L / 2$ (16 blocks) then $\operatorname{dim}(P) \approx 48 L^{3}$, to be compared to $V=12 L^{4}$ if we were to consider the full Dirac operator.

The reduction of $\bar{N}_{1}$ alone turns out not to be sufficient to make stochastic acceptance-rejection steps like in Eq. (4.1), with the Schur complement ratio, efficient. Moreover the relative gauge fixing described in Section 4.2 does not directly help in reducing the stochastic noise in this case. The reason is that the restriction of the gauge transformations to the black points in Fig. 11 leaves the Schur complement invariant. This is why the gauge field interpolation is necessary to further reduce the noise. As we show in the next section relative gauge fixing has an impact on the interpolation.

\subsection{Numerical results}

The interpolated fields $U_{i}$ in Eq. (4.16) change if we apply first a relative gauge fixing of $U$ and $U^{\prime}$, which minimizes their distance in the sense of Eq. (4.8). In Fig. 2 we show the behavior of the plaquette of the interpolated fields $U_{i}$. In the computation of the plaquette, if $U$ denotes a link then the link in reversed direction is defined by $U^{\dagger}$ (and not by $U^{-1}$ ). Without relative gauge fixing the intermediate configurations look like if they were thermalized configurations of a smaller $\beta$. 


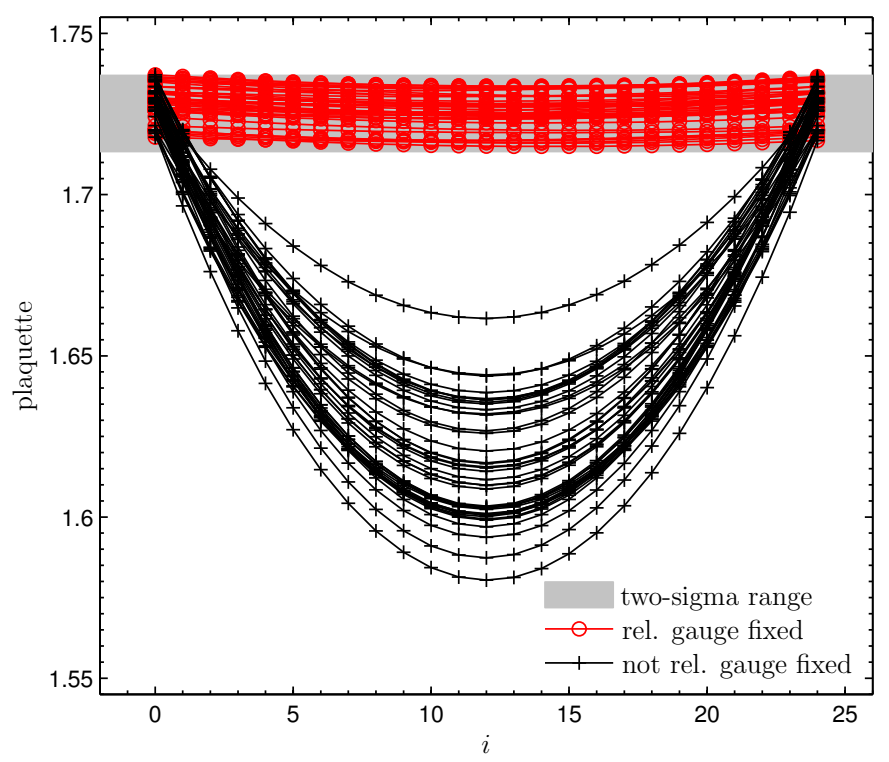

Figure 2: The plaquette value for the interpolated fields $U_{i}$ defined in Eq. (4.16) is shown as a function of $i$. The start and end fields $U_{0}$ and $U_{N}(N=24,40$ pairs $)$ are $8^{4}$ gauge configurations taken from simulations of plain Wilson fermions at $\beta=5.6$, $\kappa=0.15825$, where active links in $4^{4}$ blocks are changed. We compare plaquette values with (red circles) and without (black pluses) relative gauge fixing.

The links become rougher. This is understandable if one imagines that the gauge configurations $U$ and $U^{\prime}$ lay somewhere randomly in the configuration space. So the path will not go over configurations which are similar to the "thermalized" ones. With relative gauge fixing, the path of the interpolated links yields plaquette values which are approximately constant, cf. Fig. 2 which also shows the twosigma band of a thermalized ensemble. In Fig. 3 we show the spectra of the Schur complement ratios $M_{i}^{\dagger} M_{i}$ in Eq. (4.14). Since relative gauge fixing is applied to all links the spectrum is narrower and the requirement $\lambda>0.5$ can be fulfilled for a relatively low value of interpolation steps $N$. As expected from the behavior of the plaquette the width of the spectrum does not change significantly along the interpolation.

There are many possible ways to define alternative interpolations replacing Eq. (4.16). For example we could normalize the links by substituting $U_{i}(x, \mu) \rightarrow$ $U_{i}(x, \mu) \operatorname{det}\left(U_{i}(x, \mu)\right)^{-1 / 3}$. It turns out that in this case the intermediate configurations look like if they were thermalized configurations at larger $\beta$. As a consequence the spectrum can develop negative eigenvalues for small quark masses. We note that a mass-shift towards larger masses can be generated by multiplying the 


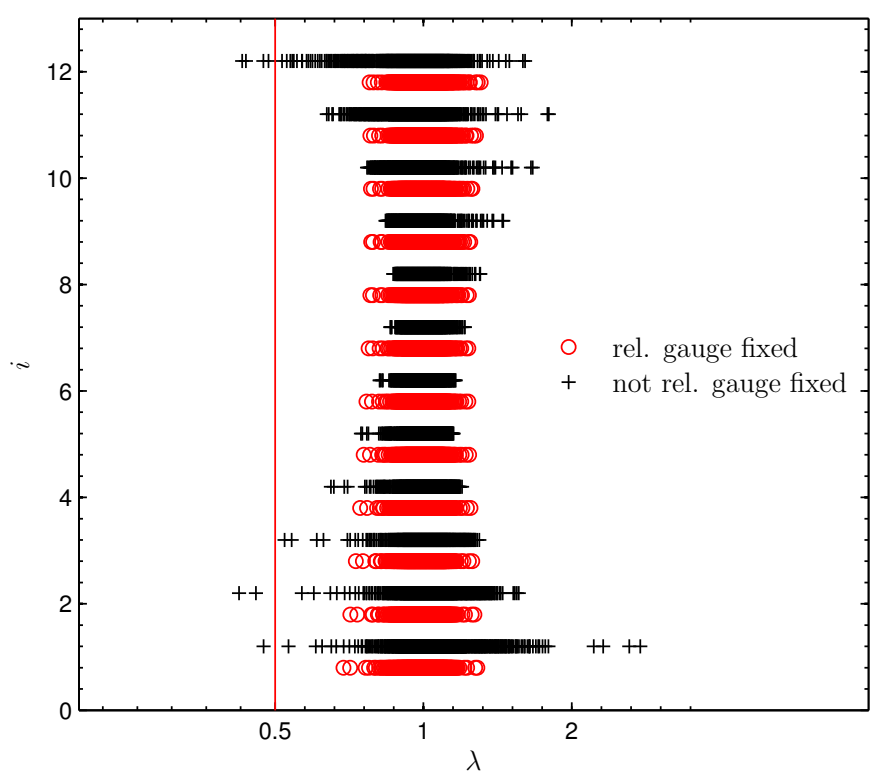

Figure 3: The spectrum of the Schur complement ratio $M_{i}^{\dagger} M_{i}$ defined in Eq. (4.14) is shown as a function of $i$. The start and end fields $U_{0}$ and $U_{N}(N=12)$ are quenched $4^{4}$ lattices where all links are changed (and relative gauge fixed). The plain WilsonDirac operator with mass $a m_{0}=0.56$ is used and the Schur complement is defined for a domain decomposition in $2^{4}$ blocks.

links with a common factor $\exp (\alpha), \alpha<0$, in the Dirac operator. Effectively such a factor (albeit with a different value for each link) can be easily incorporated into Eq. (4.16) by multiplying the links $U_{i}(x, \mu)$ with an appropriate power of their determinant $\operatorname{det}\left(U_{i}(x, \mu)\right)$. If we do not normalize the links, a "mass shift" towards larger masses is automatically realized because $\operatorname{det}\left(U_{i}(x, \mu)\right)<1$. But there is some room for improving the efficiency of the method. In the following we will use the simple interpolation given in Eq. (4.16).

Finally we discuss what happens if the relative gauge fixing is extended to the entire lattice and is not restricted to the points inside the blocks. Links which are unchanged after the pure gauge update would change through a global minimization. This could introduce additional noise and indeed this is the case for the full Dirac operator but not for the Schur complement. The global minimization slightly improves the behavior of the interpolated fields in Eq. (4.16) but this effect is not large and the danger to run into negative eigenvalues as discussed above increases. 


\begin{tabular}{|c|c|c|c|c|c|}
\hline$\imath$ & $n_{i}$ & $S^{(0)}=S_{\mathrm{w}}$ & $\begin{array}{c}\text { actions } \\
S^{(1)}=S_{\mathrm{w}}^{\mathrm{HYP}}\end{array}$ & $S^{(2)}=S_{b}$ & $P_{\text {acc }}$ \\
\hline 0 & 500 & $\beta_{0}^{(0)}=5.6918$ & - & 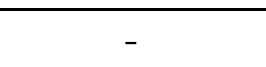 & - \\
\hline 1 & 1 & $\beta_{1}^{(0)}=-0.0196$ & $\beta_{1}^{(1)}=0.0963$ & - & $95 \%$ \\
\hline 2 & 1 & $\beta_{2}^{(0)}=-0.1187$ & $\beta_{2}^{(1)}=-0.0614$ & $\beta_{2}^{(2)}=1.644$ & $76 \%$ \\
\hline 3 & 1 & $\beta_{3}^{(0)}=0.0465$ & $\beta_{3}^{(1)}=-0.0349$ & $\beta_{3}^{(2)}=-0.644$ & varies \\
\hline
\end{tabular}

Table 1: Optimal parameters for the 4-step PSMS algorithm (representative set) for plain Wilson fermions at $\beta=5.6$ and $\kappa=0.15825$.

\section{Volume dependence of the exact acceptance rate}

We simulate QCD with $N_{\mathrm{f}}=2$ flavors of mass-degenerate quarks. The action for the gauge field is the Wilson plaquette gauge action [28]

$$
S_{g}=\beta S_{\mathrm{w}}(U)=\frac{\beta}{6} \sum_{p} \operatorname{Re} \operatorname{Tr}\{1-U(p)\},
$$

where $p$ runs over all oriented plaquettes (i.e., each plaquette is counted with two orientations). For the fermions we use the plain Wilson-Dirac operator [28] (without clover term and without smearing) with bare quark mass $m_{0}$, whose action on a quark field $\psi$ is given by

$$
\begin{aligned}
& \left(D_{\mathrm{w}}(U)+m_{0}\right) \psi(x)=\left(4+m_{0}\right) \psi(x)- \\
& \sum_{\mu=0}^{3} \frac{1}{2}\left\{U(x, \mu)\left(1-\gamma_{\mu}\right) \psi(x+\hat{\mu})+U(x-\hat{\mu}, \mu)^{\dagger}\left(1+\gamma_{\mu}\right) \psi(x-\hat{\mu})\right\}
\end{aligned}
$$

The hopping parameter is defined as $\kappa=1 /\left(2 m_{0}+8\right)$. In this section we simulate at parameters $\beta=5.6$ and $\kappa=0.15825$. Theses values corresponds to a lattice spacing $a=0.0717(15) \mathrm{fm}\left[35\right.$ and a pseudoscalar mass $m_{\mathrm{PS}} \approx 404 \mathrm{MeV}$ [36] (determined on a larger $32 \times 24^{3}$ lattice).

We implement a 4-step PSMS algorithm based on a domain decomposition with block size $4^{4}$ and on a hierarchy of three acceptance-rejection steps. Our code is based on the freely available software package DD-HMC by M. Lüscher [31]. In order to enhance the acceptance rates we introduce parameters as explained in Appendix C.

In the first step we update the active links in the $4^{4}$ blocks, which amount to a fraction of about $9.4 \%$ of all links. The gauge proposal consists of 500 iterations of two Cabibbo-Marinari heat-bath [37] sweeps (with reversed sequence of 

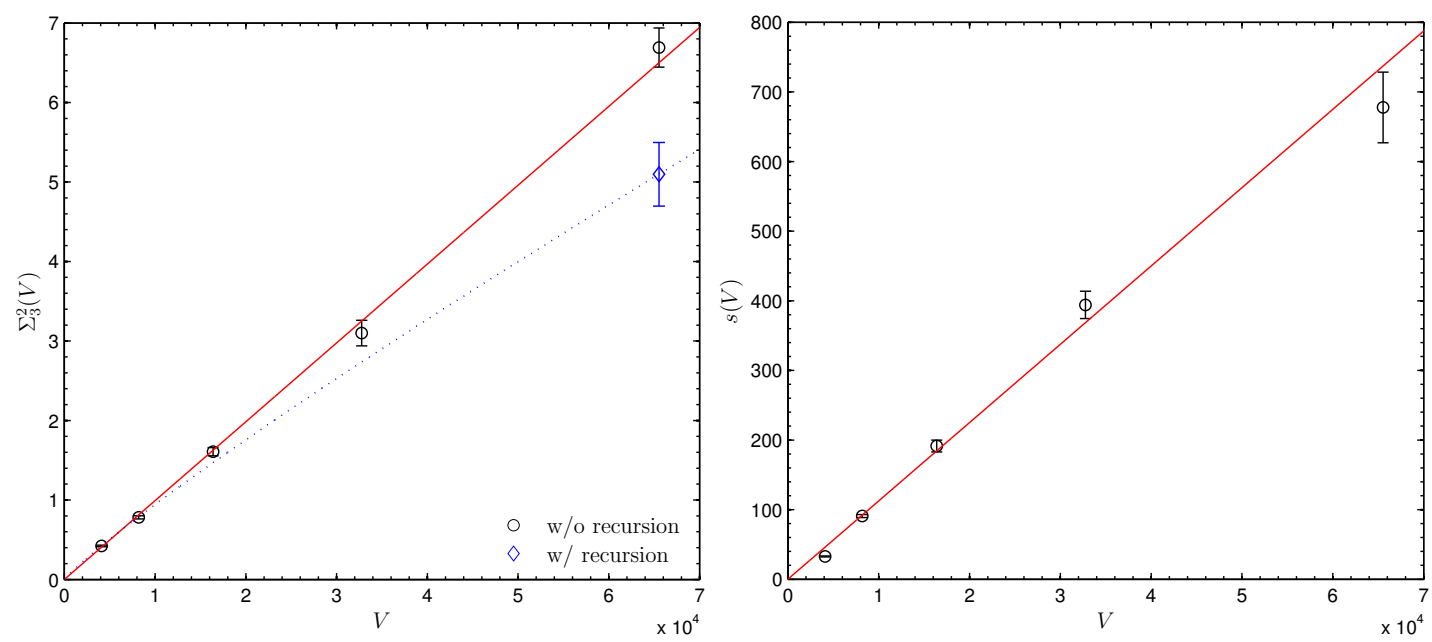

Figure 4: The variance of the stochastic estimator in the global step from simulations of plain Wilson fermions at $\beta=5.6, \kappa=0.15825$ with the 4-step PSMS algorithm. The left plot shows the exact variance $\Sigma_{3}^{2}$ (black circles) as a function of the lattice volume $V$ together with a linear fit (red line). The blue diamond is the result using a 5-step PSMS algorithm, see text. The right plot shows the volume dependence of the slope $s(V)$ defined in Eq. (5.6) together with a linear fit.

gauge link updates and random choice of $\mathrm{SU}(2)$ subgroups) at the shifted coupling $\beta_{0}^{(0)}=5.6918$. The gauge proposal is then subjected to a first acceptance-rejection step containing a plaquette action $S_{\mathrm{w}}^{\mathrm{HYP}}$ like in Eq. (5.1) but where the plaquettes are constructed from HYP smeared links with the parameters of [32] (one level of smearing). We do one iteration of this step with $95 \%$ acceptance. The resulting proposal goes into a second acceptance-rejection step containing the action $S_{b}=\sum_{b \in \mathcal{C}} 2 \ln \left(\operatorname{det}\left(D_{b}\right)\right)$ of the block determinants (one iteration with $76 \%$ acceptance). We emphasize that the first and second acceptance-rejection (or filter) steps are done block-wise and can be therefore parallelized. Finally the gauge proposal which passed through the first two filter steps enters the global acceptancerejection step with the Schur complement of the $4^{4}$ block decomposition. This is a stochastic acceptance-rejection step performed according to Eq. (4.15) using the interpolation with intermediate fields $U_{i}, i=0, \ldots, N$ in Eq. (4.16). The optimal parameters can be tuned following the prescription given in Section C.2. We note that they depend only mildly on the global lattice volume and a representative set is listed in Table 1 .

The global acceptance-rejection probability is $P_{\text {acc }}^{(3)}=\min \left\{1, \exp \left(-\Delta_{3}\right)\right\}$, 
where (cf. Eq. (C.2))

$$
\Delta_{3}=\beta_{3}^{(0)} \Delta S_{\mathrm{w}}+\beta_{3}^{(1)} \Delta S_{\mathrm{w}}^{\mathrm{HYP}}+\beta_{3}^{(2)} \sum_{b \in \mathcal{C}} 2 \Delta \ln \left(\operatorname{det}\left(D_{b}\right)\right)+\sum_{i=0}^{N-1} \eta_{i}^{\dagger}\left(M_{i}^{\dagger} M_{i}-1\right) \eta_{i}
$$

and $M_{i}$ is the ratio of Schur complements. On lattices with $V=8^{4}$ up to $V=$ $16^{4}$ we measure, for different values of the gauge field interpolation steps $N$ in Eq. (4.13), the variance

$$
\sigma_{3}^{2}(V, N)=\left\langle\left(\Delta_{3}-\left\langle\Delta_{3}\right\rangle\right)^{2}\right\rangle_{U, U^{\prime}, \eta}
$$

At fixed volume $V$ we extrapolate linearly in $1 / N$ to zero, thus obtaining an estimate for the exact variance $\Sigma_{3}^{2}(V)$ as a function of the volume. The justification for this extrapolation is given by Eq. (4.3), which in this case means

$$
\sigma_{3}^{2}(V, N)=\Sigma_{3}^{2}(V)+\left(\sigma_{3, N}^{\text {stoch }}(V)\right)^{2}
$$

and by Eq. (4.17), which implies

$$
\left(\sigma_{3, N}^{\text {stoch }}(V)\right)^{2} \approx \frac{1}{N} s(V)
$$

with the slope $s(V)$ is approximately given by $\bar{N}_{1} h_{1}^{2}$. Here $\bar{N}_{1}$ and $h_{1}$ refer to the Schur complement ratio. Note that $\sigma_{3}^{2}(V, N)$ contains also contributions from parts of the action other than the Schur complement (cf. Eq. (C.5)) but which do not depend on $N$. The extrapolated exact variance $\Sigma_{3}^{2}(V)$ is shown in the left plot of Fig. 4 as a function of $V$. The data can be very well fitted by a straight line constrained to zero at zero volume (red line). The slopes $s(V)$ of the linear fits of $\sigma_{3}^{2}(V, N)$ in $1 / N$ are plotted against the volume $V$ in the right plot of Fig. 4. The data of the slope can be also well fitted by a straight line constrained to zero at zero volume (red line). Assuming that $\bar{N}_{1}$ is equal to the dimension of the projector $P$ in Eq. (4.20) and taking into account that the block size is here constant and equal to $4^{4}$, we deduce that $\bar{N}_{1} \propto V$. Therefore our results for the slope means that the FWHM $h_{1}$ of the generalized eigenvalues of the Schur complements does not significantly depend on the volume.

Via (2.6) the exact acceptance rate can be determined 8 from the variance $\Sigma_{3}^{2}(V)$. The exact acceptance rates as determined from the variances are plotted in Fig. 5 (black circles) together with the result from the fit to $\Sigma_{3}^{2}(V)$ shown in the left plot of Fig. 4. The 4-step PSMS algorithm of this section shows a good acceptance for lattices up to $16^{3} \times 8$. This is the region where the error

\footnotetext{
${ }^{8}$ We tested the (tacitly assumed) validity of the Gaussian model for finite values of $N$.
} 


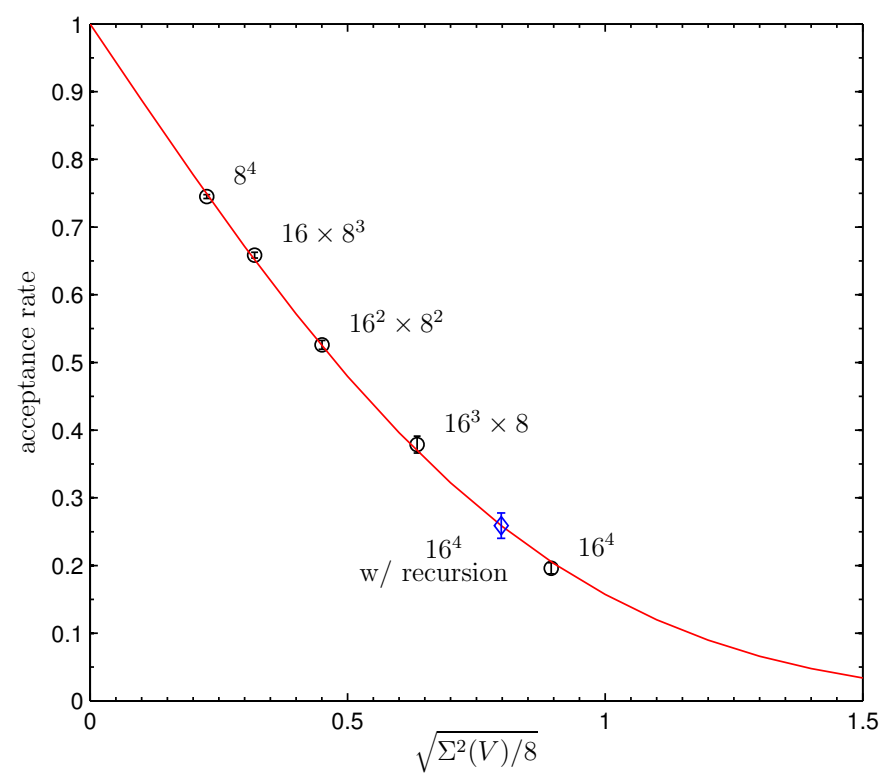

Figure 5: The exact global acceptance is plotted as a function of the variance $\Sigma^{2}$ for simulations of plain Wilson fermions at $\beta=5.6, \kappa=0.15825$ with the 4-step PSMS algorithm (black circles). The point corresponding to the blue diamond is from a simulation of a $16^{4}$ lattice with a 5 -step PSMS algorithm.

function can be approximated by a Taylor expansion with a linear leading term $\operatorname{erfc}(x)=1-2 x / \sqrt{\pi}+\mathrm{O}\left(x^{3}\right)$. Fig. 9 shows that the acceptance rates, which one would obtain from the Schur complement alone (blue diamonds), are much smaller.

The efficiency of the hierarchy of filters in enhancing the acceptance of the global step can be demonstrated by simulating the largest $16^{4}$ lattice using a 5 step PSMS algorithm. For this we use a recursive domain decomposition of the $16^{4}$ lattice in $8^{4}$ and $4^{4}$ blocks, cf. Eq. (3.3). The additional filter with respect to the 4-step PSMS algorithm is a stochastic acceptance-rejection step accounting for the Schur complements of the $4^{4}$ blocks within the $8^{4}$ blocks, cf. Eq. (3.4). The acceptance of the global step (accounting for the global Schur complement) is increased by this further filter step, cf. the blue diamond in Fig. [5. Using recursive domain decomposition to keep the largest block size at $L / 2$ (where $V=L^{4}$ ), the volume dependence of $\Sigma^{2}$ in the global step is $V^{q}$ (dotted line in the left plot of Fig. (4) with $q \approx 0.9$ (determined on our available lattices $8^{4}$ and $16^{4}$ ). At large $V$ one expects the asymptotic behavior $q=3 / 4$, cf. Section 4.4 , 


\begin{tabular}{|c|c|c|c|c|c|c|}
\hline$i$ & $n_{i}$ & $N_{i}$ & $S^{(0)}=S_{\mathrm{w}}$ & $\begin{array}{c}\text { actions } \\
S^{(1)}=S_{\mathrm{w}}^{\mathrm{HYP}}\end{array}$ & $S^{(2)}=S_{b}$ & $P_{\mathrm{acc}}$ \\
\hline 0 & 75 & - & $\beta_{0}^{(0)}=5.9822$ & - & - & - \\
\hline 1 & 3 & - & $\beta_{1}^{(0)}=-0.0378$ & $\beta_{1}^{(1)}=0.2110$ & - & $69 \%$ \\
\hline 2 & 3 & 96 & $\beta_{2}^{(0)}=-0.1376$ & $\beta_{2}^{(1)}=-0.2110$ & $\beta_{2}^{(2)}=1.0711$ & $48 \%$ \\
\hline 3 & 1 & 96 & $\beta_{3}^{(0)}=-0.0068$ & $\beta_{3}^{(1)}=0$ & $\beta_{3}^{(2)}=-0.0711$ & $64 \%$ \\
\hline
\end{tabular}

Table 2: Parameters for the 4-step PSMS algorithm for plain Wilson fermions at $\beta=5.8$ and $\kappa=0.15462$.

\section{Numerical tests of the algorithm}

We present results of simulations of $N_{\mathrm{f}}=2$ flavors of mass-degenerate plain Wilson fermions on a $16^{4}$ lattice at $\beta=5.8$ and $\kappa=0.15462$. The clover coefficient is set to zero and the fermions have anti-periodic boundary conditions in time direction. The lattice spacing is estimated in [35] to be $0.0521(7) \mathrm{fm}$ and the pseudoscalar mass is $381 \mathrm{MeV}$ [36] (determined on a larger $64 \times 32^{3}$ lattice).

In the simulations in Section 5 our smallest blocks are $4^{4}$ and the gauge proposal changes the active links in these blocks. It turns out that larger blocks are better in terms of changing the topological charge and allow for higher global acceptances (at somewhat higher computational cost). That is why we change our setup in this section and use $8^{4}$ blocks as our smallest ones. The gauge proposal changes the active links in a $6^{4}$ hypercube inside each of the $8^{4}$ blocks, which amounts to updating $7.9 \%$ of all gauge links.

We adopt a 4-step PSMS algorithm whose parameters and acceptances are summarized in Table 2. For each of the steps $i=0,1,2,3, n_{i}$ is the number of iterations per step and $N_{i}$ is the number of gauge field interpolation steps (for stochastic estimates of Schur complement ratios). The gauge proposal consists of a number $n_{0}$ of iterations of symmetrized sweeps of Cabibbo-Marinari heatbath [37] and over-relaxation [38, 39] updates. One iteration consists of one heatbath (HB) sweep and $L / 2$ over-relaxation (OR) sweeps followed by the reversed sequence of link updates (so in total one $\mathrm{HB}+L / 2 \mathrm{OR}+L / 2 \mathrm{OR}+$ one $\mathrm{HB}$ sweeps), where for each link we choose with probability $1 / 2$ one sequence of $\mathrm{SU}(2)$ subgroups and with probability $1 / 2$ the reversed sequence. The first acceptancerejection step is a Metropolis step for a HYP plaquette gauge action which has to be subtracted in the successive filter steps. The determinant of the $8^{4}$ blocks is factorized by a domain decomposition in $4^{4}$ blocks. The second acceptancerejection step accounts for the exact product of the $4^{4}$ block determinants times the 


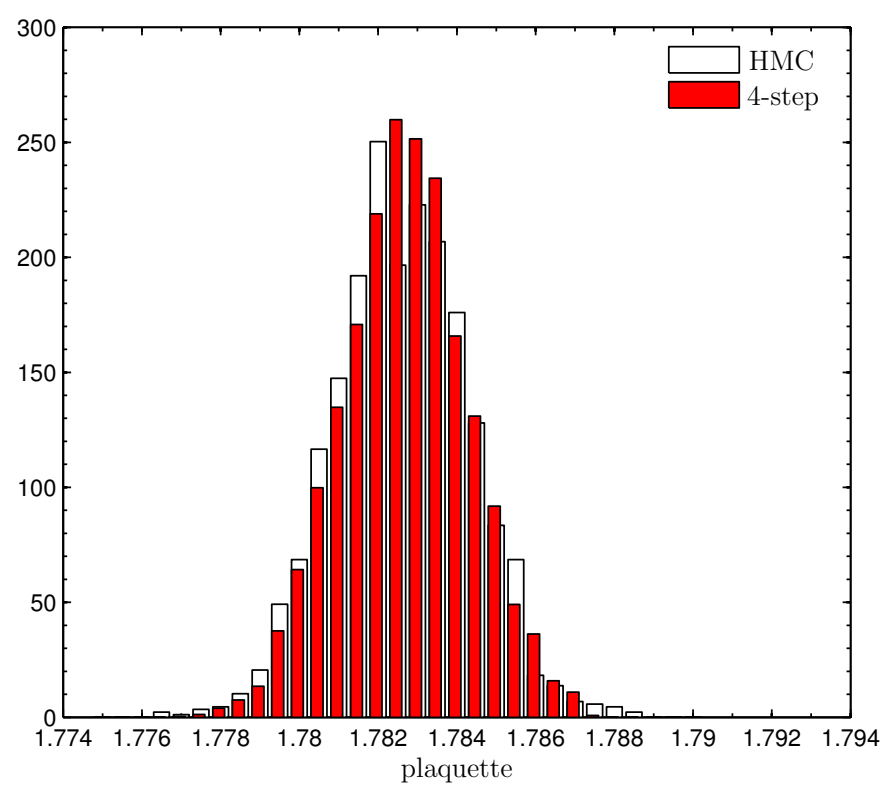

Figure 6: Histogram distribution of the plaquette values from simulations of plain Wilson fermions and Wilson plaquette action at $\beta=5.8, \kappa=0.154620,16^{4}$ lattices. We compare results for the 4-step PSMS algorithm and for the HMC.

determinant of the Schur complement of the decomposition of the $8^{4}$ blocks in $4^{4}$ blocks. The latter is treated stochastically. These two acceptance-rejection steps are performed independently for each $8^{4}$ block. The third stochastic acceptancerejection step contains the global Schur complement of the decomposition of the $16^{4}$ lattice in $8^{4}$ blocks.

In Fig. 6] we show the histogram distribution of the plaquette value. We compare the results from 4 replica simulated using the 4-step PSMS algorithm (red bins) with the results from a long HMC simulation (white bins). The HMC simulation is done with the DD-HMC algorithm [21, 40] using $8^{4}$ blocks. The distributions agree perfectly.

In the upper two plots of Fig. 7 the histories of the topological charge are shown. The topological charge is defined by

$$
Q=\frac{1}{16 \pi^{2}} \sum_{x, \mu, \nu} F_{\mu \nu}(x) \tilde{F}_{\mu \nu}(x),
$$

using a discretization of the field strength tensor $F_{\mu \nu}$ (see e.g. [30]) in which gauge links constructed from three levels of HYP smearing are used. We consider 4 replica simulated using the 4 -step PSMS algorithm (left plot) and 3 replica simulated with the HMC (right plot). The horizontal dotted lines are determined 

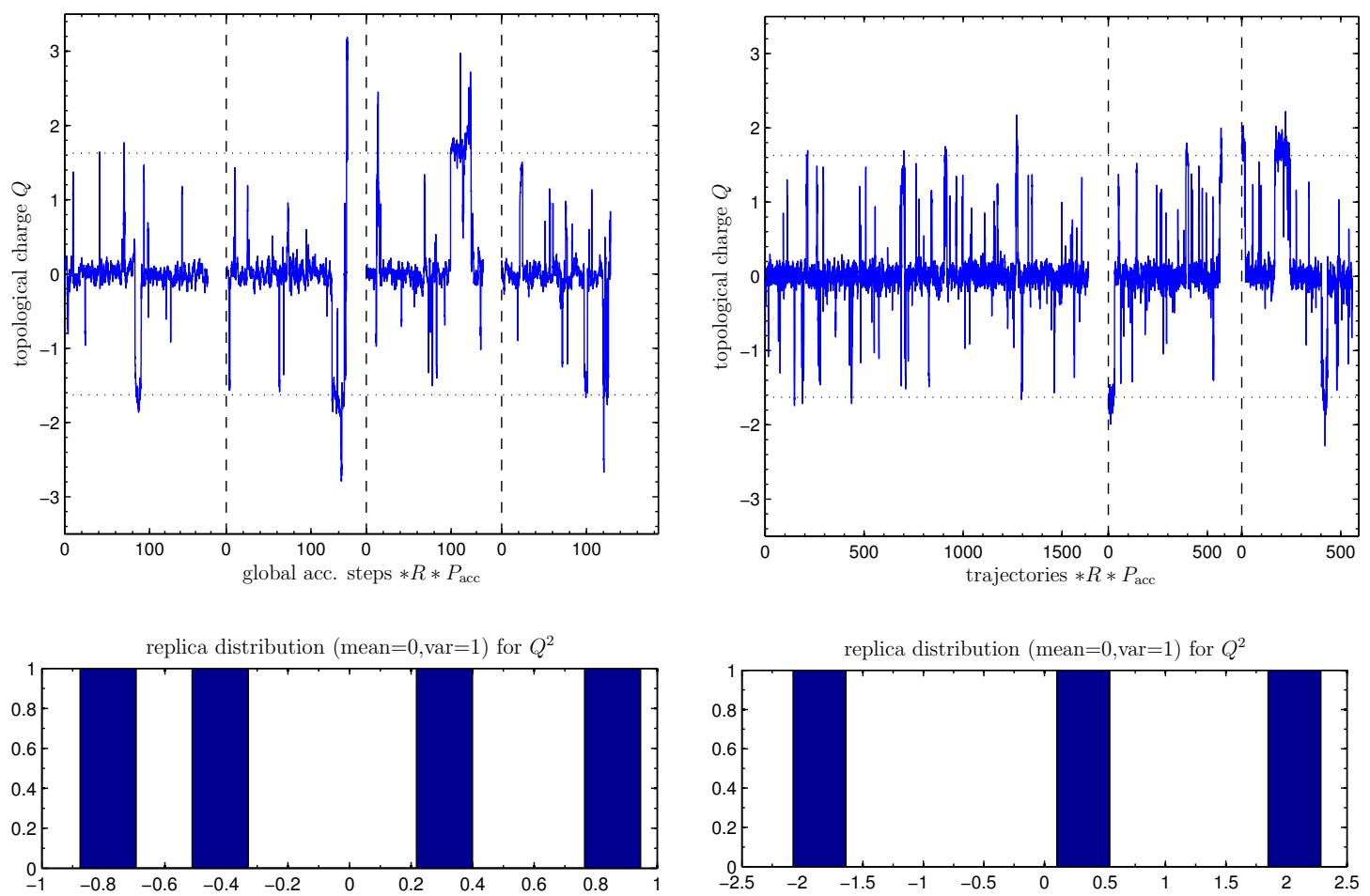

Figure 7: Histories of the topological charge $Q$ (upper plots) and histograms of the deviations of the replicum means of $Q^{2}$ from the total mean divided by the replicum errors (lower plots). The left plots show the results of 4 replica simulated with the 4-step PSMS algorithm. The right plots show the HMC results from 3 replica.

from an ad hoc fit to the histogram of the topological charge using 3 Gaussian functions (one centered at zero and the other at values $\pm m$ corresponding to the dotted lines). In order to compare the Monte Carlo histories of the two algorithms, we take the Monte Carlo units which correspond to a full change of the gauge configuration. To this end, on the x-axis of the history plots we take, for the PSMS algorithm, the number of global acceptance steps multiplied by the fraction $R$ of links changed and by the global acceptance while, for the HMC algorithm, we take the number of trajectories multiplied by the ratio $R$ of active links and by the acceptance. The right plot shows that the long HMC replicum was not able to really tunnel to a topological sector different than zero, while such a tunneling occurred at least once for all PSMS replica. Indeed we compared the distributions of the topological charge squared $Q^{2}$ for the PSMS replica and the long HMC replicum and found that they agree well around $Q^{2}=0$ but differ at larger values. Therefore we started two more HMC replica from configurations with topological charge different than zero (generated in the PSMS ensembles), which are also shown in Fig. 17. In one of these two additional replica we observe 

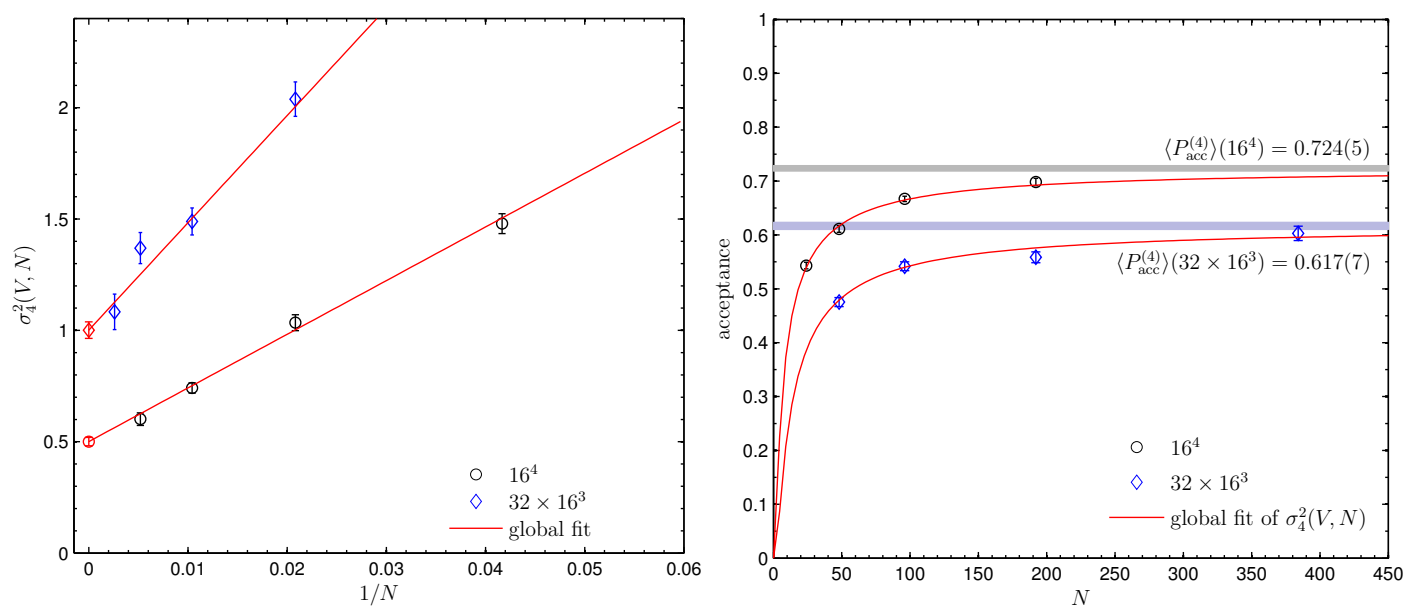

Figure 8: The variance $\sigma_{3}^{2}(V, N)$ (left plot) in the global step for simulations of plain Wilson fermions and Wilson plaquette action at $\beta=5.8, \kappa=0.154620$. Data for $V=16^{4}$ (circles) and $V=32 \times 16^{3}$ (diamonds) are very well fitted as functions of $1 / N$ using a global linear fit (red lines). In the right plot we show the resulting acceptances.

a clear tunneling from topological sector zero to nonzero. In the lower two plots of Fig. 7 we show histograms of the deviations of the replicum means of $Q^{2}$ from the total mean divided by the replicum errors (the quantity in Eq. (30) of [41]; left plot, PSMS; right plot, HMC). The goodness of the replica distribution is measured by the probability (goodness-of-fit) of a constant fit to the replicum means. The goodness is 0.7 for the PSMS algorithm and 0.05 for the HMC. A value much below 0.1 is very unlikely. The expecation value $\left\langle Q^{2}\right\rangle$ is $0.37(15)$ for the 4-step PSMS algorithm and 0.281(81) for the HMC algorithm. The errors are determined using the method of [41]. From leading order chiral perturbation theory we expect $\left\langle Q^{2}\right\rangle \approx 0.19$. We emphasize that Fig. 7 is a comparison made at one lattice spacing only. The main problem is the scaling with the lattice spacing which we cannot address in the scope of this paper.

In Fig. 8 we plot the variance $\sigma_{3}^{2}(V, N)$ (left plot) and the acceptance (according to Eq. (2.6), right plot) in the global acceptance-rejection step (see Eq. (5.5)) as a function of $1 / N$ and $N$ respectively. Together with the data for the $16^{4}$ lattices we present data for $32 \times 16^{3}$ lattices. Motivated by the results of Section 5 we perform a global fit to the variances of the form

$$
\sigma_{3}^{2}(V, N)=V\left(a_{1}+\frac{a_{2}}{N}\right)
$$

with fit parameters $a_{1}$ and $a_{2}$. The exact variance turns out to be $\Sigma_{3}^{2}=0.50(2)$ and $\Sigma_{3}^{2}=1.00(4)$ for the $16^{4}$ and $32 \times 16^{3}$ lattices respectively. This corresponds 
to acceptances $0.724(5)$ and $0.617(7)$.

A cost comparison of the simulations of the $16^{4}$ lattice can be performed by comparing the number of full inversions of the Wilson-Dirac operator needed to update all the links. Using the 4-step PSMS algorithm at optimal parameters, the global acceptance-rejection step has $N=96$ gauge field interpolation steps (each of which requires one inversion of the full operator for the inversion of the global Schur complement, see Eq. (4.19) ) and 64\% acceptance. This means that we need $\approx 23$ global steps or 2200 inversions to get a new gauge configuration. If we instead run the 4-step PSMS algorithm with $N=24$ and $42 \%$ global acceptance, one new gauge configuration is obtained after $\approx 35$ global steps or 840 inversions. The DD-HMC needs only 120 inversions for one new gauge configuration. This naive cost comparison does not take into account effects of autocorrelation times, which are hard to estimate for observables like the topological charge.

\section{Conclusions}

We have developed and tested the PSMS algorithm for lattice QCD that consists of a hierarchical filter of acceptance-rejection steps. The hierarchy is based on an exact factorization of the fermion determinant. Although other factorization are possible, we here deploy (recursive) domain decomposition as it separates the determinant in a local (blocks) and global part (Schur complement).

We were able to determine the exact global acceptance rates for volumes up to $(1.2 \mathrm{fm})^{4}$ and demonstrate that the filter is successful in fighting the exponential decrease with the volume.

The global acceptance-rejection step with the Schur complement remains expensive. We estimate a factor of ten in comparison with the HMC for the setup of Section 6. The expected scaling of the cost of the algorithm with the volume is

$$
V(\text { inversion }) \times V^{3 / 4}(N) \times 1 /(\text { acceptance }) .
$$

The first factor is due to the cost of one inversion of the Dirac operator and the second factor arises from the necessity to keep the stochastic noise low. A constant global acceptance is achieved for constant variance $\Sigma^{2}$ of the action differences that go into the global step, i.e., $\sigma_{\hat{\lambda}}^{2} \propto 1 / V$ is needed (cf. Eq. (2.11)). Instead we find $\sigma_{\hat{\lambda}}^{2} \sim$ const as $V$ is increased (cf. Fig. 4). Previously the fluctuations of the small eigenvalues of $\sqrt{D^{\dagger} D}$ have been found to decrease like $1 / V$ [26]. We do not seem to see this behavior for $\sigma_{\hat{\lambda}}^{2}$. The reason might be that our separation scale, given by the inverse block size $1 / L_{\mathrm{b}}$, is too large. For the simulations at $\beta=5.8$ $(\beta=5.6)$ with a block size of 8 this scale is approximately $500 \mathrm{MeV}(360 \mathrm{MeV})$. 
At the moment the performance of the PSMS algorithm is worse than the one of the HMC algorithm, but the scaling of autocorrelation times of the topological charge with the lattice spacing has to be studied to make a definite conclusion. In Fig. 7 we present evidence that the PSMS algorithm is more efficient in sampling the topological sectors compared to the HMC. It is still relevant to study alternatives to the HMC and there are prospects of using and improving the PSMS algorithm. One possibility is to apply reweighting for the Schur complement, cf. 42] where we demonstrate that reweighting factors for the Schur complement have a better scaling with the volume compared to the full operator. Improved gauge actions can be included in the hierarchy of acceptance steps and there is room for better choices of the gauge updates within the blocks. Also factorizations of the determinant other than domain-decomposition could be used.

The techniques for the stochastic estimation of determinant ratios, which we introduced in this article for the acceptance-rejection steps, can be equally well applied to the case of reweighting, e.g., in the quark mass [42] or to account for electromagnetic effects.

Acknowledgement. We thank Tony Kennedy for correspondence on the proof of detailed balance, Martin Lüscher for a clarification on the fluctuations of small eigenvalues of the Dirac operator, Rainer Sommer for discussions and Ulli Wolff for comments on the relative gauge fixing. The Monte Carlo simulations were carried out on the cluster Stromboli at the University of Wuppertal and we thank the University.

\section{A Proofs of detailed balance}

\section{A.1 Exact acceptance-rejection steps}

The simplest setup of our algorithm is to split up the gauge weight in Eq. (2.7) from the fermionic one. The idea is to propose a new gauge configuration $U^{\prime}$ by a pure gauge updating algorithm and accept or reject it by a Metropolis step accounting for the fermionic weight. Let $T_{0}\left(U \rightarrow U^{\prime}\right)$ be the transition probability for the pure gauge proposal which has to satisfy detailed balance for the distribution (see below)

$$
P_{0}(U)=\frac{\exp \left(-S_{g}(U)\right)}{Z_{0}},
$$

where $Z_{0}$ is the partition function for the gauge action $S_{g}$. The Metropolis acceptance-rejection step [23] consists of accepting or rejecting the proposal $U^{\prime}$ 
with probability

$$
P_{\text {acc }}\left(U, U^{\prime}\right)=\min \left\{1, \frac{P_{0}(U) P\left(U^{\prime}\right)}{P(U) P_{0}\left(U^{\prime}\right)}\right\} .
$$

The transition probability for this algorithm is

$$
\begin{aligned}
& T\left(U \rightarrow U^{\prime}\right)=T_{0}\left(U \rightarrow U^{\prime}\right) P_{\mathrm{acc}}\left(U, U^{\prime}\right) \\
& +\delta\left(U-U^{\prime}\right)\left(1-\int D\left[U^{\prime \prime}\right] T_{0}\left(U \rightarrow U^{\prime \prime}\right) P_{\mathrm{acc}}\left(U, U^{\prime \prime}\right)\right) .
\end{aligned}
$$

In order for $T$ to satisfy detailed balance for the distribution $P$ in Eq. (2.7), $T_{0}$ has to satisfy detailed balance for the distribution $P_{0}$ in Eq. (A.1). If the gauge proposal is a sequence of gauge link updates, their order has to be symmetrized or chosen randomly [20].

\section{A.2 Stochastic acceptance-rejection steps}

The exact calculation of the determinant ratio in Eq. (A.2) is numerically prohibitive. It can be replaced by a stochastic approximation that maintains detailed balance exactly [13].

We follow closely Appendix A, in particular section A.5, of [22]. The variables of the system (the gauge field) are enlarged by adding auxiliary stochastic variables, which are called pseudofermions and are only used in the stochastic acceptance-rejection step. The equilibrium probability distribution for the enlarged system of gauge field $U$ and pseudofermion $\eta$ is

$$
\hat{P}(\eta, U)=\frac{\mathrm{e}^{-\left|D(U)^{-1} \eta\right|^{2}} \exp \left(-S_{g}(U)\right)}{Z} .
$$

The pseudofermion is a complex-valued field $\eta$ with the measure

$$
D[\eta]=\prod_{x, \alpha} \frac{d \operatorname{Re}\left(\eta_{x, \alpha}\right) d \operatorname{Im}\left(\eta_{x, \alpha}\right)}{\pi}
$$

where the index $\alpha$ contains spin and color degrees of freedom. The norm squared of $\eta$ is defined by the scalar product $(\eta, \eta)$ :

$$
|\eta|^{2}=(\eta, \eta)=\sum_{x, \alpha} \eta_{x, \alpha}^{*} \eta_{x, \alpha}
$$

The equilibrium distribution of the gauge field alone is recovered by integrating over the pseudofermion:

$$
P(U)=\int D[\eta] \hat{P}(\eta, U)
$$


We also define the conditional probability

$$
\hat{P}(\eta \mid U)=\frac{\hat{P}(\eta, U)}{P(U)}=\frac{\mathrm{e}^{-\left|D(U)^{-1} \eta\right|^{2}}}{|\operatorname{det} D(U)|^{2}}
$$

to generate the pseudofermion field $\eta$ given the gauge field $U$.

The algorithm to update the enlarged system consists of alternating two Markov steps. The first is a global heatbath step for updating the pseudofermion at given gauge field $U$. A new pseudofermion $\eta$ distributed according to $\hat{P}(\eta \mid U)$ in Eq. (A.8) is generated through

$$
\eta=D(U) \xi
$$

where $\xi$ is a Gaussian random pseudofermion generated with probability distribution $p(\xi)=\exp \left(-|\xi|^{2}\right)$ 9. The second step is a Metropolis step for the gauge field at given pseudofermion. A new gauge field $U^{\prime}$ is proposed with transition probability $T_{0}\left(U \rightarrow U^{\prime}\right)$, which satisfies detailed balance for the distribution $P_{0}(U)$ in Eq. (A.1). The proposal is followed by an acceptance-rejection step with probability

$$
\min \left\{1, \frac{P_{0}(U) \hat{P}\left(\eta, U^{\prime}\right)}{\hat{P}(\eta, U) P_{0}\left(U^{\prime}\right)}\right\}=\min \left\{1, \frac{\mathrm{e}^{-\left|D\left(U^{\prime}\right)\right|^{2} \eta}}{\mathrm{e}^{-|D(U)|^{2} \eta}}\right\} .
$$

Both the heatbath and Metropolis steps separately fulfill detailed balance with respect to the combined probability distribution $\hat{P}(\eta, U)$ in Eq. (A.4) [20]. Therefore also their composition has the correct fixed point probability [43].

We consider now a composite update step consisting of an heatbath update for the pseudofermion in Eq. (A.9) immediately followed by a Metropolis step for the gauge field in Eq. (A.10). If after this we forget the pseudofermion field, this can be viewed as an update for the gauge field alone with acceptance probability 10

$$
\begin{aligned}
P_{\text {acc }}\left(U, U^{\prime}\right) & =\int D[\eta] \hat{P}(\eta \mid U) \min \left\{1, \frac{P_{0}(U) \hat{P}\left(\eta, U^{\prime}\right)}{\hat{P}(\eta, U) P_{0}\left(U^{\prime}\right)}\right\} \\
& =\int D[\xi] \mathrm{e}^{-|\xi|^{2}} \min \left\{1, \mathrm{e}^{-|M \xi|^{2}+|\xi|^{2}}\right\},
\end{aligned}
$$

where the ratio operator $M$ is defined in Eq. (2.10). The associated transition probability in Eq. (A.3), where now $P_{\text {acc }}\left(U, U^{\prime}\right)$ is given by Eq. (A.11), satisfies detailed balance for the equilibrium probability $P(U)$ due to the property [22]

$$
\left[P(U) / P_{0}(U)\right] P_{\text {acc }}\left(U, U^{\prime}\right)=\left[P\left(U^{\prime}\right) / P_{0}\left(U^{\prime}\right)\right] P_{\text {acc }}\left(U^{\prime}, U\right),
$$

\footnotetext{
${ }^{9}$ The pseudofermion measure in Eq. A.5 is normalized such that $\int D[\xi] p(\xi)=1$.

${ }^{10}$ We thank Tony Kennedy for clarifying this point in a correspondence.
} 
or equivalently [13]

$$
\frac{P_{\mathrm{acc}}\left(U, U^{\prime}\right)}{P_{\mathrm{acc}}\left(U^{\prime}, U\right)}=|\operatorname{det}(M)|^{-2} .
$$

In practice, the acceptance step Eq. (A.11) is done by drawing one Gaussian distributed pseudofermion $\xi$ and accepting or rejecting depending on the argument under the min function. We note that it is not possible to perform the average of the argument under the min function over many pseudofermions, as this violates detailed balance. The acceptance probability in Eq. (A.11) was computed in [13]

$$
P_{\mathrm{acc}}\left(U, U^{\prime}\right)=\sum_{i} \min \left(1,1 / \lambda_{i}\right) \prod_{j \neq i} \frac{\lambda_{i}-1}{\lambda_{i}-\lambda_{j}}
$$

in terms of the eigenvalues $\lambda_{i}$ of $M^{\dagger} M$. It is bounded by the exact (non-stochastic) acceptance probability in Eq. (A.2) [13]

$$
P_{\text {acc }}\left(U, U^{\prime}\right) \leq \min \left\{1,|\operatorname{det}(M)|^{-2}\right\} .
$$

So far we discussed the case of a proposal followed by an acceptance-rejection steps. Eq. (A.2) can be generalized to an arbitrary number of acceptance-rejection steps as discussed in Section 2. The algorithm satisfies detailed balance and this is also true if (some of) the Metropolis acceptance-rejections steps are replaced by their stochastic counterpart Eq. (A.11).

\section{A.3 Gauge field interpolation}

In order to simplify a bit the notation we consider an algorithm like it is described in Section A.2 with one stochastic acceptance-rejection step. In practice we apply the gauge field interpolation method to acceptance-rejection steps involving the Schur complements (the global Schur complement $\hat{D}$ as well as the Schur complement in the blocks $\hat{D}_{b}$ when we use recursive domain decomposition, see Eq. (3.3) ).

For the gauge proposal $U \rightarrow U^{\prime}$ we consider the gauge field interpolation $U_{i}$ as it is given in Eq. (4.16). For each of the transitions $U_{i} \rightarrow U_{i+1}, i=0,1, \cdots, N-1$ we introduce a pseudofermion field $\eta_{i}$. The equilibrium probability distribution for the enlarged system is

$$
\hat{P}\left(\left\{\eta_{j}\right\}, U, U^{\prime}\right)=\frac{\mathrm{e}^{-\left|D\left(U^{g}\right)^{-1} \eta_{0}\right|^{2}} \mathrm{e}^{-S_{g}(U)}}{Z} \prod_{i=1}^{N-1} \frac{\mathrm{e}^{-\left|D\left(U_{i}\right)^{-1} \eta_{i}\right|^{2}}}{\left|\operatorname{det}\left(D\left(U_{i}\right)\right)\right|^{2}}
$$


and depends now also on the proposed configuration $U^{\prime}$. Integrating over the pseudofermions gives

$$
P(U)=\int \prod_{i=0}^{N-1} D\left[\eta_{i}\right] \hat{P}\left(\left\{\eta_{j}\right\}, U, U^{\prime}\right)
$$

The conditional probability to generate the pseudofermions $\left\{\eta_{j}\right\}$ given the proposal $U \rightarrow U^{\prime}$ is

$$
\hat{P}\left(\left\{\eta_{j}\right\} \mid U, U^{\prime}\right)=\frac{\hat{P}\left(\left\{\eta_{j}\right\}, U, U^{\prime}\right)}{P(U)}=\prod_{i=0}^{N-1} \frac{\mathrm{e}^{-\left|D\left(U_{i}\right)^{-1} \eta_{i}\right|^{2}}}{\left|\operatorname{det}\left(D\left(U_{i}\right)\right)\right|^{2}}
$$

We use the property $\operatorname{det}(D(U))=\operatorname{det}\left(D\left(U^{g}\right)\right)$.

If we consider the reversed gauge proposal $U^{\prime} \rightarrow U$ (i.e. $U_{0}=U^{\prime g^{-1}}$ and $U_{N}=U^{g}$ ), the intermediate configurations $U_{i}$ in Eq. (4.16) are the same but they are traversed in reversed order and therefore the pseudofermion $\eta_{i}$ is associated with the transition $U_{i+1} \rightarrow U_{i}$. The probability distribution for the enlarged system is now

$$
\hat{P}\left(\left\{\eta_{j}\right\}, U^{\prime}, U\right)=\frac{\mathrm{e}^{-\left|D\left(U^{\prime g}{ }^{-1}\right)^{-1} \eta_{N-1}\right|^{2}} \mathrm{e}^{-S_{g}\left(U^{\prime}\right)}}{Z} \prod_{i=0}^{N-2} \frac{\mathrm{e}^{-\left|D\left(U_{i+1}\right)^{-1} \eta_{i}\right|^{2}}}{\left|\operatorname{det}\left(D\left(U_{i+1}\right)\right)\right|^{2}}
$$

and the conditional probability to generate the pseudofermions $\left\{\eta_{j}\right\}$ is

$$
\hat{P}\left(\left\{\eta_{j}\right\} \mid U^{\prime}, U\right)=\frac{\hat{P}\left(\left\{\eta_{j}\right\}, U^{\prime}, U\right)}{P\left(U^{\prime}\right)}=\prod_{i=0}^{N-1} \frac{\mathrm{e}^{-\left|D\left(U_{i+1}\right)^{-1} \eta_{i}\right|^{2}}}{\left|\operatorname{det}\left(D\left(U_{i+1}\right)\right)\right|^{2}} .
$$

The acceptance probability for the gauge proposal $U \rightarrow U^{\prime}$ is

$$
\begin{aligned}
P_{\text {acc }}\left(U, U^{\prime}\right) & =\int \prod_{i=0}^{N-1} D\left[\eta_{i}\right] \hat{P}\left(\left\{\eta_{j}\right\} \mid U, U^{\prime}\right) \min \left\{1, \frac{P_{0}(U) \hat{P}\left(\left\{\eta_{j}\right\}, U^{\prime}, U\right)}{\hat{P}\left(\left\{\eta_{j}\right\}, U, U^{\prime}\right) P_{0}\left(U^{\prime}\right)}\right\} \\
& =\int \prod_{i=0}^{N-1} D\left[\xi_{i}\right] \mathrm{e}^{-\left|\xi_{i}\right|^{2}} \min \left\{1, \mathrm{e}^{\sum_{j=0}^{N-1}-\left|M_{j} \xi_{j}\right|^{2}+\left|\xi_{j}\right|^{2}}\right\},
\end{aligned}
$$

where

$$
M_{i}=D\left(U_{i+1}\right)^{-1} D\left(U_{i}\right) .
$$

$P_{\text {acc }}\left(U, U^{\prime}\right)$ in Eq. (A.21) fulfills the detailed balance condition Eq. (A.12) or equivalently

$$
\frac{P_{\mathrm{acc}}\left(U, U^{\prime}\right)}{P_{\mathrm{acc}}\left(U^{\prime}, U\right)}=|\operatorname{det}(M)|^{-2}, \quad M=D\left(U^{\prime}\right)^{-1} D(U) .
$$


In practice, the global acceptance step Eq. (A.21) is done by drawing $N$ Gaussian distributed pseudofermions $\xi_{i}$ and accepting or rejecting depending on the argument of the min function (i.e. we evaluate the sum in the exponent under the min function).

\section{B Relative gauge fixing}

The relative gauge fixing of two gauge field configurations $U$ and $U^{\prime}$ is done using a steepest descent scheme introduced by [44,45] for gauge group SU(3). Using the condition Eq. (4.12) for the gauge transformation $g$, the minimization condition in Eq. (4.9) can be written similarly to the case of the Landau gauge condition. Then one can apply the procedure of [45] to fix the relative gauge.

At each point $x$ where the gauge transformation $g$ is defined we have to solve the condition

$$
\min _{g(x)} \operatorname{Re} \operatorname{Tr}\left\{1-\left(g(x)^{\dagger}\right)^{2} \cdot\left(W_{\mathrm{f}}(x)+W_{\mathrm{b}}(x)\right\}\right.
$$

where

$$
\begin{aligned}
& W_{\mathrm{f}}(x)=\sum_{\mu} U^{\prime}(x, \mu) g(x+\hat{\mu})^{2} U^{\dagger}(x, \mu), \\
& W_{\mathrm{b}}(x)=\sum_{\mu} U^{\prime \dagger}(x-\hat{\mu}, \mu) g(x-\hat{\mu})^{2} U(x-\hat{\mu}, \mu) .
\end{aligned}
$$

Using the steepest descent method of [45] we get the minimizing transformation field $g(x)$ iteratively through

$$
g(x)=\exp \left\{-\frac{\alpha}{2}\left[\Delta-\Delta^{\dagger}-\frac{1}{3} \operatorname{Tr}\left(\Delta-\Delta^{\dagger}\right)\right]\right\}
$$

with a scaling parameter $\alpha$ and

$$
\Delta=W_{\mathrm{f}}(x)+W_{\mathrm{b}}(x) .
$$

The minimum is reached when $\theta(x)=0$, where

$$
\theta(x)=\operatorname{Tr}\left[\Delta-\Delta^{\dagger}-\frac{1}{3} \operatorname{Tr}\left(\Delta-\Delta^{\dagger}\right)\right]^{2} .
$$

We choose the value $\alpha=0.15$. If there is no convergence we reduce it in steps of -0.01 and reach values down to $\alpha=0.10$. For the $\mathrm{SU}(3)$ exponential function in Eq. (B.4) we use the matrix function described in Appendix A of [21]. The numerical cost of the relative gauge fixing can be reduced in the case of a domain 


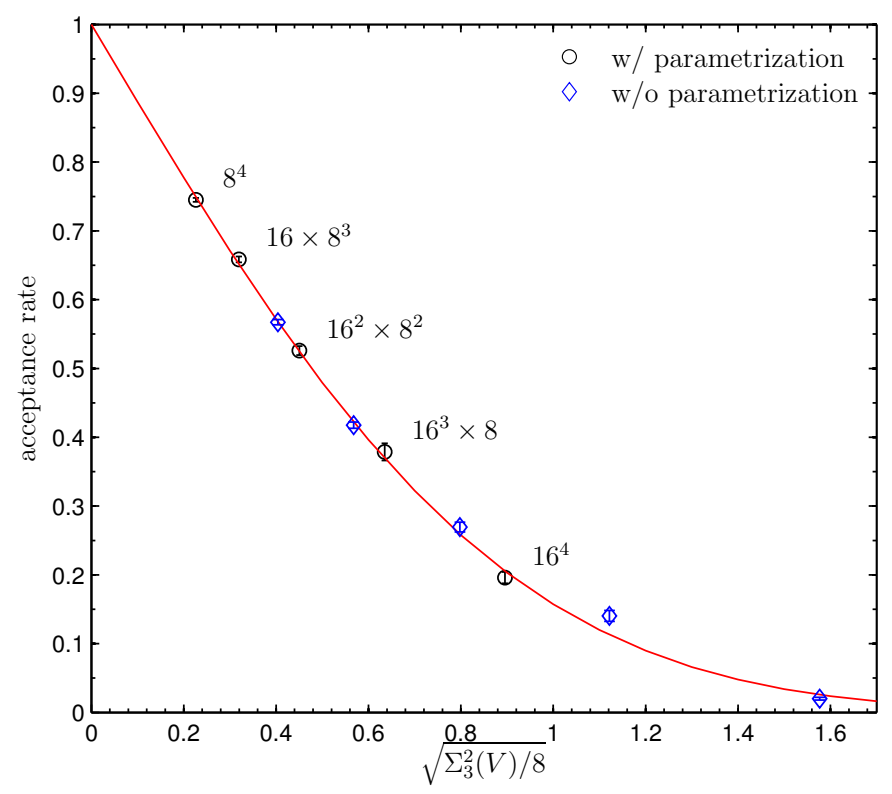

Figure 9: The exact global acceptance is plotted as a function of the variance $\Sigma_{3}^{2}$ for simulations of plain Wilson fermions at $\beta=5.6, \kappa=0.15825$ with the 4-step PSMS algorithm. The black circles are the same as in Fig. [5] and corresponds to the optimal acceptance. The blue diamonds represent the exact acceptance which one would get from the Schur complement alone without the additional parameters (last row in Table 1).

decomposition by defining $g(x)$ only inside the blocks where the active links are changed. Further it can be reduced by stopping the iteration when $\theta(x)<10^{-3}$, which we find good enough for the purpose of the gauge field interpolation discussed in Section 4.3.

\section{Parametrized acceptance-rejection steps}

The general idea of the PSMS algorithm is to factorize the distribution in Eq. (2.7) in several pieces and introduce a recursive update procedure with a computational cost ordering. Naively speaking a gauge configuration is proposed by a pure gauge algorithm and the fermion determinant is treated in acceptance-rejection steps. It is easy to see that the plaquette gauge action and the determinant of the Dirac operator are strongly correlated. This correlation can be used to increase the acceptance [13, 14, 24] and also in the case of reweighting [46]. This is an example of ultraviolet filtering [20,47,48]. 


\section{C.1 Optimization of the acceptance}

We consider the hierarchy of acceptance-rejection steps $i=1,2, \ldots, n$ in Eq. (2.3). The factorization (2.2) is not unique and can be parametrized. In each acceptancerejection step the action involved might be written as

$$
S_{i}=\sum_{j=0}^{i} \beta_{i}^{(j)} S^{(j)}, \quad i=1, \ldots, n,
$$

with real coefficients $\beta_{i}^{(j)}$ and actions $S^{(j)}$. The probability to accept the proposal for a new gauge field $U^{\prime}$ starting from $U$ is $\min \left\{1\right.$, $\left.\exp \left(-\Delta_{i}\right)\right\}$ where

$$
\Delta_{i}=S_{i}\left(U^{\prime}\right)-S_{i}(U)=\sum_{j=0}^{i} \beta_{i}^{(j)} \Delta S^{(j)} .
$$

For Gaussian distributed $\Delta_{i}$ the acceptance rate is given by erfc $\left(\sqrt{\Sigma_{i}^{2} / 8}\right)$ with

$$
\Sigma_{i}^{2}=\left\langle\left(\Delta_{i}-\left\langle\Delta_{i}\right\rangle\right)^{2}\right\rangle .
$$

In the case of the factorization Eq. (3.2), $n=2$ and $S^{(0)}$ is the gauge action, $S^{(1)}=\sum_{b \in \mathcal{C}} 2 \ln \left(\operatorname{det}\left(D_{b}\right)\right)$ and $S^{(2)}=2 \ln (\operatorname{det}(\hat{D}))$ are the effective actions of the determinants of the blocks and of the Schur complement respectively. In stochastic acceptance-rejection steps, like we do for the Schur complement, we use

$$
\Delta S^{(2)}=\eta^{\dagger}\left(M^{\dagger} M-1\right) \eta,
$$

where $\eta$ is a Gaussian noise vector and $M=\hat{D}\left(U^{\prime}\right)^{-1} \hat{D}(U)$. In such case the variance $\Sigma_{i}^{2}$ in Eq. (C.3) is replaced by the sum of the exact variance and the stochastic variance according to Eq. (4.3). The variance in Eq. (C.3) can be written explicitly in terms of the coefficients as

$$
\Sigma_{i}^{2}=\sum_{j=0}^{i}\left(\beta_{i}^{(j)}\right)^{2} C^{(j j)}+\sum_{\substack{j, k=0 \\ j \neq k}}^{i} \beta_{i}^{(j)} \beta_{i}^{(k)} C^{(j k)},
$$

where $C^{(j k)}=\left\langle\left(\Delta S^{(j)}-\left\langle\Delta S^{(j)}\right\rangle\right)\left(\Delta S^{(k)}-\left\langle\Delta S^{(k)}\right\rangle\right)\right\rangle$. We note that here $\langle\cdot\rangle$ means an average over configurations $U$ in the dynamical ensemble and over gauge proposals $U^{\prime}$ (and over noise $\eta$ if applicable).

At each step $i=1, \ldots, n$ the optimization of the parameters $\beta_{i}^{(j)}$ is done by minimizing the variance $\Sigma_{i}^{2}$ in Eq. (C.3). The idea is to use the correlation 
of low cost actions with the high cost action of the $i$ th step to increase the $i$ thlevel acceptance rate. In order to get the right distribution after the last step the parameters of a specific action $S^{(j)}$ has to sum up to the target value $\beta^{(j)}=\sum_{i} \beta_{i}^{(j)}$. This implies constraints on the parameter. For example the parameter of the plaquette action has to sum up to $\beta^{(0)}=\beta$. In principle, in order to solve for the parameters, we can start from the last step $i=n$, solve for the parameters $\beta_{n}^{(j)}$ and go to step $i-1$. This provides an explicit solution scheme. At step $i$, we solve the linear system of $i$ equations

$$
2 C^{(j j)} \beta_{i}^{(j)}+\sum_{\substack{k=0 \\ k \neq j}}^{i-1} C^{(j k)} \beta_{i}^{(k)}=-C^{(j i)} \beta_{i}^{(i)}, \quad j=0, \ldots, i-1,
$$

to uniquely determine the values of the coefficients $\beta_{i}^{(0)}, \ldots, \beta_{i}^{(i-1)}$. The solutions of the steps $k>i$ and one constraint imply $\beta_{i}^{(i)}=\beta^{(i)}-\sum_{k=i+1}^{n} \beta_{k}^{(i)}$.

We emphasize some properties of the parametrized acceptance-rejection steps. First of all this quite simple technique guarantees that the distribution of the pure gauge proposal has a good overlap with the dynamical distribution. Without parametrization the acceptance rate for lattices bigger than $4^{4}$ would be less than few $\%$. The parametrization introduces a $\beta$-shift to higher $\beta$ values in the pure gauge update, mainly reflecting the correlation with the determinants on the small blocks, see Table 1 and Table 2, In general it is possible to introduce a new acceptance-rejection step $i$ by defining an auxiliary action with additional parameters. These parameters have to sum up to zero when considering all acceptance-rejection steps $k \geq i$. Their effect is to enhance the acceptance rate of these steps. For example we introduced a plaquette action, which uses HYP smeared links (one level of smearing) in order to better match the pure gauge update with the fermionic weight. This is particularly motivated for simulations with HYP smeared Wilson fermions but also helps for plain Wilson fermions, see Table 1 and Table 2. We remark that it is not possible to introduce parameters for terms which are evaluated stochastically like Eq. (C.4 $)$. The effectiveness of the parametrization of acceptance-rejection steps is demonstrated in Fig. 9, where we compare the exact acceptances in the global step with optimal parameters (black circles) to the exact acceptances without parameters (blue diamonds).

\section{C.2 Tuning the optimal parameters}

The parameters in the acceptance-rejection steps $i=1,2, \ldots, n-1$ are estimated from a simulation where the global step $i=n$ (the computationally most costly) is left out. Subsequently a full simulation is performed in order to determine the 
optimal parameters for the global step. Iterating further this procedure does not significantly change the values of the parameters.

\section{References}

[1] S. Duane, A. Kennedy, B. Pendleton and D. Roweth, Phys.Lett. B195 (1987) 216.

[2] S.A. Gottlieb, W. Liu, D. Toussaint, R. Renken and R. Sugar, Phys.Rev. D35 (1987) 2531.

[3] ALPHA Collaboration, S. Schaefer, R. Sommer and F. Virotta, Nucl.Phys. B845 (2011) 93, 1009.5228.

[4] L. Del Debbio, H. Panagopoulos and E. Vicari, JHEP 0208 (2002) 044, hep-th/0204125.

[5] M. Lüscher, JHEP 1008 (2010) 071, 1006.4518.

[6] M. Lüscher and S. Schaefer, JHEP 1107 (2011) 036, 1105.4749.

[7] L. Del Debbio, L. Giusti, M. Lüscher, R. Petronzio and N. Tantalo, JHEP 0602 (2006) 011, hep-lat/0512021.

[8] S. Dürr et al., JHEP 1108 (2011) 148, 1011.2711.

[9] A. Hasenfratz, R. Hoffmann and S. Schaefer, JHEP 0705 (2007) 029, heplat/0702028.

[10] C. Morningstar and M.J. Peardon, Phys.Rev. D69 (2004) 054501, heplat/0311018.

[11] S. Capitani, S. Dürr and C. Hoelbling, JHEP 0611 (2006) 028, heplat/0607006.

[12] W. Kamleh, D.B. Leinweber and A.G. Williams, Phys.Rev. D70 (2004) 014502, hep-lat/0403019.

[13] Alpha Collaboration, F. Knechtli and U. Wolff, Nucl.Phys. B663 (2003) 3, hep-lat/0303001.

[14] A. Hasenfratz and F. Knechtli, Comput.Phys.Commun. 148 (2002) 81, heplat/0203010. 
[15] A. Hasenfratz and A. Alexandru, Nucl.Phys.Proc.Suppl. 119 (2003) 994, hep-lat/0209071.

[16] A. Hasenfratz, Nucl.Phys.Proc.Suppl. 119 (2003) 131, hep-lat/0211007.

[17] A. Hasenfratz, P. Hasenfratz and F. Niedermayer, Phys.Rev. D72 (2005) 114508, hep-lat/0506024.

[18] B. Joo, I. Horvath and K. Liu, Phys.Rev. D67 (2003) 074505, heplat/0112033.

[19] J. Finkenrath, F. Knechtli and B. Leder, (2011), 1112.1243.

[20] M. Hasenbusch, Phys.Rev. D59 (1999) 054505, hep-lat/9807031.

[21] M. Lüscher, Comput.Phys.Commun. 165 (2005) 199, hep-lat/0409106.

[22] M. Lüscher, JHEP 0305 (2003) 052, hep-lat/0304007.

[23] N. Metropolis, A. Rosenbluth, M. Rosenbluth, A. Teller and E. Teller, J.Chem.Phys. 21 (1953) 1087.

[24] A.C. Irving and J.C. Sexton, Phys.Rev. D55 (1997) 5456, hep-lat/9608145.

[25] D. Weingarten and D. Petcher, Phys.Lett. B99 (1981) 333.

[26] M. Lüscher and F. Palombi, PoS LATTICE2008 (2008) 049, 0810.0946.

[27] M. Hasenbusch, Phys. Lett. B519 (2001) 177, hep-lat/0107019.

[28] K.G. Wilson, Phys.Rev. D10 (1974) 2445.

[29] B. Sheikholeslami and R. Wohlert, Nucl.Phys. B259 (1985) 572.

[30] M. Lüscher, S. Sint, R. Sommer and P. Weisz, Nucl.Phys. B478 (1996) 365, hep-lat/9605038.

[31] M. Lüscher, http://luscher.web.cern.ch/luscher/DD-HMC/index.html.

[32] A. Hasenfratz and F. Knechtli, Phys.Rev. D64 (2001) 034504, heplat/0103029.

[33] T.A. Davis, http://www.cise.ufl.edu/research/sparse/umfpack/.

[34] A. Hasenfratz and A. Alexandru, Phys.Rev. D65 (2002) 114506, heplat/0203026. 
[35] L. Del Debbio, L. Giusti, M. Luscher, R. Petronzio and N. Tantalo, JHEP 0702 (2007) 056, hep-lat/0610059, TeX source, 17 pages, figures included.

[36] L. Del Debbio, L. Giusti, M. Luscher, R. Petronzio and N. Tantalo, JHEP 0702 (2007) 082, hep-lat/0701009.

[37] N. Cabibbo and E. Marinari, Phys.Lett. B119 (1982) 387.

[38] S.L. Adler, Phys.Rev. D23 (1981) 2901.

[39] R. Petronzio and E. Vicari, Phys.Lett. B245 (1990) 581.

[40] M. Lüscher, JHEP 0712 (2007) 011, 0710.5417.

[41] ALPHA collaboration, U. Wolff, Comput.Phys.Commun. 156 (2004) 143, hep-lat/0306017.

[42] J. Finkenrath, F. Knechtli and B. Leder, (2012), 1211.1214.

[43] A. Kennedy, (2006), hep-lat/0607038.

[44] G.G. Batrouni et al., Phys. Rev. D32 (1985) 2736.

[45] C.T.H. Davies et al., Phys. Rev. D37 (1988) 1581.

[46] A. Hasenfratz, R. Hoffmann and S. Schaefer, Phys.Rev. D78 (2008) 014515, 0805.2369.

[47] P. de Forcrand, Nucl.Phys.Proc.Suppl. 73 (1999) 822, hep-lat/9809145.

[48] A. Duncan, E. Eichten, R. Roskies and H. Thacker, Phys.Rev. D60 (1999) 054505, hep-lat/9902015. 